TRANSACTIONS OF THE

AMERICAN MATHEMATICAL SOCIETY

Volume 364, Number 7, July 2012, Pages 3627-3655

S 0002-9947(2012)05617-0

Article electronically published on March 7, 2012

\title{
GREEN FUNCTIONS FOR A CLASS OF NONLINEAR DEGENERATE OPERATORS WITH X-ELLIPTICITY
}

\author{
SHENZHOU ZHENG AND ZHAOSHENG FENG
}

Abstract. A maximum principle and some a priori estimates of a class of degenerate equations with X-ellipticity in the sense of distributions are established. A local comparison of the generalized Green function with its fundamental solutions is obtained. As an application, by means of the power of the Green function as a kernel function of a local integral, we also derive local Hölder continuity for nonlinear degenerate subelliptic equations.

\section{INTRODUCTION}

Let $\Omega$ be a bounded open subset of $\mathbb{R}^{N}$ for $N \geq 3$. Given a family of vector fields $X=\left(X_{1}, \cdots, X_{m}\right)$, we assume that each component $X_{k}=\left(b_{k 1}, b_{k 2}, \cdots, b_{k n}\right)$ : $\Omega \rightarrow \mathbb{R}^{N}$ is locally Lipschitz continuous for $k=1,2, \cdots, m$. We identify the vector field $X_{k}$ with $X_{k} u=\left\langle X_{k}, \nabla u\right\rangle=\sum_{j=1}^{N} b_{k j} \partial_{j} u$ if $u \in C^{1}(\Omega)$, and when $u \in L_{l o c}^{1}(\Omega)$ it is understood in the distributional sense that

$$
X_{k} u=\sum_{j=1}^{N} \partial_{x_{j}}\left(b_{k j} u\right)-\left(\sum_{j=1}^{N} \partial_{x_{j}}\left(b_{k j}\right)\right) u,
$$

for $k=1,2, \cdots, m$.

In this paper, we consider the nonlinear degenerate elliptic operator of divergence form:

$$
\mathcal{A} u:=-\operatorname{div}\left\{\langle A(x) \nabla u, \nabla u\rangle^{\frac{p-2}{2}} A(x) \nabla u\right\}, \quad x \in \Omega,
$$

where $p>1$, each entry of the bounded measurable coefficient matrix $A(x)=$ $\left(a_{i j}(x)\right)$ satisfies $a_{i j}(x) \in L^{\infty}(\Omega)$ and $a_{i j}(x)=a_{j i}(x)$. We suppose that the operator $\mathcal{A}$ satisfies the following uniform X-ellipticity in $\Omega$ :

$$
\frac{1}{\lambda} \sum_{k=1}^{m}\left\langle X_{k}(x), \xi\right\rangle^{2} \leq\langle A(x) \xi, \xi\rangle \leq \lambda \sum_{k=1}^{m}\left\langle X_{k}(x), \xi\right\rangle^{2}, \quad \forall x \in \Omega, \quad \forall \xi \in \mathbb{R}^{N},
$$

where $\lambda$ is a positive constant and $\langle\cdot, \cdot\rangle$ denotes the standard inner product in $\mathbb{R}^{N}$. The notion of X-ellipticity was implicitly introduced in 21 by Franchi and Lanconelli in 1982, after which it was intensively studied in a series of works

Received by the editors July 27, 2010.

2010 Mathematics Subject Classification. Primary 35J70, 35H20, 35D10.

Key words and phrases. Green function, X-ellipticity, control distance, maximum principle, Morrey's space, hole-filling technique.

This work was supported by NSF (China) Grant No.11071012 and UTPA Faculty Research Council Grant 145MATH04.

The second author was the corresponding author. 
2, 3, 6, 7, 9, 13, 16, 17, 22, 27, 28, etc. In 2000, it was explicitly developed in [40] by Lanconelli and Kogoj. From the uniform X-ellipticity assumption (1.2), a direct calculation shows

$$
\frac{1}{\lambda}|X \varphi|^{2} \leq\langle A(x) \nabla \varphi, \nabla \varphi\rangle \leq \lambda|X \varphi|^{2} .
$$

Since $|\langle A(x) \xi, \eta\rangle| \leq\langle A(x) \xi, \xi\rangle^{\frac{1}{2}}\langle A(x) \eta, \eta\rangle^{\frac{1}{2}}, \forall \xi, \eta \in \mathbb{R}^{N}$, from (1.2) again, we obtain

$$
|\langle A(x) \nabla \varphi, \nabla \psi\rangle| \leq \lambda|X \varphi||X \psi|,
$$

when $\varphi$ and $\psi \in C^{1}(\Omega)$.

The prototype of the nonlinear degenerate operator (1.1) originates from the following subelliptic operators:

$$
-\Delta_{p}^{X} u:=-\sum_{k=1}^{m} X_{k}^{*}\left(|X u|^{p-2} X_{k} u\right),
$$

where $X_{i}^{*}$ is the formal adjoint of $X_{i}$ given by $X_{k}^{*} v=-\partial_{j}\left(b^{k j} v\right)$. The operator $\Delta_{p}^{X}$ corresponds to the functional

$$
J_{p}(u)=\int_{\Omega}|X u|^{p} d x
$$

which arises naturally in the geometry of CR manifolds [34, 35, and the theory of quasi-conformal mappings on stratified and nilpotent Lie groups.

In order to recall the definition of control distance (or the so-called CarnotCarathéodory distance) associated with $\mathrm{X}$-vector fields in $\mathbb{R}^{N}$, we start with

Definition 1. An absolutely continuous path $\gamma:[0, T] \rightarrow \Omega \subset \mathbb{R}^{N}$ is said to be an X-subunit if $\dot{\gamma}(t)=\sum_{k=1}^{m} c_{k}(t) X_{k}(\gamma(t))$ with $\sum_{k=1}^{m} c_{k}(t) \leq 1$, for almost every $t \in[0, T]$.

Assume that $\Omega \subset \mathbb{R}^{N}$ is X-connected, i.e. for every $x, y \in \Omega$, there exists at least one $\mathrm{X}$-subunit path connecting $x$ and $y$, so we may define

$$
d(x, y)=\inf \left\{T>0 \mid \exists \gamma:[0, T] \rightarrow \Omega \subset \mathbb{R}^{N} \text { X-subunit with } \gamma(0)=x, \gamma(T)=y\right\}
$$

Out of the study of sub-Riemannian geometry, it appears that the definition of control distance has been widely used since the paper by Fefferman and Phong 24] in the smooth case and the papers by Franchi and Lanconelli [21, 22] in the nonsmooth case. Some basic properties of the linear X-elliptic operators together with some fundamental properties of the control distance were presented by Gutiérrez and Lanconelli [26]. For more information of control distance and related concepts and applications, we refer the reader to a recent survey paper by Hayslaz and Koskela 31 and the references therein.

By a simple calculation this shows that the nonnegative function $(x, y) \mapsto d(x, y)$ is a metric on $\Omega \subset \mathbb{R}^{N}$ [26, 43, 48]. We call $d(x, y)$ the control distance (or the Carnot-Carathéodory distance) related to $X$. Hereafter, all the distances mentioned in this context will be designated with respect to the metric $d$ unless we indicate it specifically. In particular, $B_{R}(x)$ denotes the ball $\left\{y \in \mathbb{R}^{N} \mid d(x, y)<R\right\}$ with the control metric, and $|E|$ denotes the Lebesgue measure of the set $E \subset \mathbb{R}^{N}$. Following the literature [6, 7], we define the Sobolev space for $p \geq 1$ as:

$$
W^{1, p}(\Omega, X):=\left\{u \in L^{p}(\Omega) \mid X_{k} u \in L^{p}(\Omega), k=1,2, \cdots, m\right\},
$$


equipped with the norm

$$
\|u\|_{W^{1, p}(\Omega, X)}=\left(\int_{\Omega}\left(|u|^{p}+|X u|^{p}\right) d x\right)^{\frac{1}{p}} .
$$

Now we are in a position to introduce our general assumptions of control distance $d(.,$.$) with respect to the vector fields X$ in $\mathbb{R}^{N}$ :

(H1) (Metric equivalence) If $|y-x| \rightarrow 0 \Rightarrow d(x, y) \rightarrow 0$, then we have that $|y-x| \rightarrow 0 \Leftrightarrow d(x, y) \rightarrow 0$.

(H2) (Homogeneous dimension) There are positive constants $R_{0}, C_{1}, C_{2}$ and $Q>p>1$ such that for all $R \in\left(0, R_{0}\right]$ and $x \in \Omega$, the following relation is valid:

$$
C_{1} R^{Q} \leq\left|B_{R}(x)\right| \leq C_{2} R^{Q},
$$

where the number $Q$ will be chosen as the least integer such that the above inequality holds, which is called the homogeneous dimension of $X$ in $\Omega$.

(H3) (Poincaré's inequality) There is a positive constant $C$ such that the following inequality holds:

$$
f_{B_{R}(x)}\left|u-u_{x, R}\right|^{p} d y \leq C R^{p} f_{B_{\theta R}(x)}|X u|^{p} d y, \quad \forall u \in C^{1}\left(\bar{B}_{\theta R}, X\right),
$$

where $p \geq 1, u_{x, R}=f_{B_{R}} u d x=\frac{1}{\left|B_{R}\right|} \int_{B_{R}(x)} u d x$ and $|X u|$ denotes the Euclidean norm of the generalized gradient of $u$.

From the hypothesis (H2), the following doubling property is valid [2, 31]: there is a positive constant $C$ such that for all $x \in \Omega$ and $R>0$, the following doubling condition holds:

$$
\left|B_{2 R}(x)\right| \leq C\left|B_{R}(x)\right|
$$

Again from hypothesis (H2) we have

$$
\left|B_{t R}(x)\right| \leq A t^{Q}\left|B_{R}(x)\right|, \quad t \geq 1,
$$

for all $x \in \bar{\Omega}$ and $R>0$, where $A$ is a positive constant.

The uniform X-ellipticity (1.2) deserves several comments. The subelliptic operators on a Carnot group can be viewed as particular X-elliptic operators. When the vector fields $\left\{X_{j}\right\}$ are invariant with respect to the left translations on a Carnot group $\mathbf{G}=\left(\mathbb{R}^{N}, \circ\right)$ for $p=2$, usually the operator $\mathcal{A}$ is the so-called sub-Laplacian defined on a stratified Lie group. Some fundamental and profound results of subelliptic operators have been established in $[2,14,15,16,17,12,10,11,21,22,36,40$, 46, 23, 6, 7, 4, 15, 18, 19, 44, etc., ever since the pioneering paper by Hörmander 33, where he proved that a linear subelliptic equation satisfying the Hörmander rank condition is hypoelliptic. Maximum principles and Harnack's inequalities of various subelliptic problems related to smooth and nonsmooth vector fields $\left\{X_{j}\right\}$ satisfying the rank condition "rank $\operatorname{Lie}\left[X_{1}, \cdots, X_{m}\right]=r$ at each point of the domain $\Omega$ " have been investigated in [1, 12, 18, 19, 20, 48. Various applications on Riemannian geometry of subelliptic equations are presented in the monograph by Bellaiche and Risler [3.

On the other hand, it is well known that the Green function plays an important role in various differential equations and systems, including the classical Dirichlet problem of Laplacian operators and elliptic systems, which have some perfect properties 1, 8, 38, 39, 42. It can also be applied to many problems such as Wiener's criterion for a regular boundary point of a domain [30, 32, 42, stochastic games problems 1, and the regularity of the weak solution under the weaker regular data 
[1, 49, 50. Littman and his collaborators 42, presented the comparison theory of the Green function with Laplacian operators for uniformly elliptic operators with symmetric and bounded measurable coefficients defined in $\Omega$, and gave the corresponding Wiener's criterion of regular boundary points. Grüter and Widman [30] proved the same conclusion of the Green functions and the Wiener's criterion of regular points on the boundary for more general uniformly elliptic operators with nonsymmetric coefficients. Furthermore, Negrini and Scornazzani [45] described Wiener's criterion on the boundary points related to the sum of squares of vector fields for a class of degenerate elliptic operators. By way of nonlinear potential theory and estimates of the Green function, Kilpeläinen and Malý et al. [37, 41] derived the regular tests at a boundary point of solutions of quasilinear elliptic equations and degenerate elliptic equations. Recently, Gutiérrez and Lanconelli [26] and Mazzoni [43] investigated the maximum principle, Harnack's inequality and the Green functions for linear X-elliptic operators of divergence form. Since the Green functions of various differential operators have significant applications in many scientific fields, in this paper we will show that the Green function of a class of nonlinear degenerate operators with X-ellipticity enjoys the fundamental properties analogously as the $p$-Laplacian does in Carnot groups in many respects. Here the concept of the so-called Green function for the nonlinear degenerate elliptic operator $\mathcal{A}$ with X-ellipticity is a straightforward generalization of the regular subelliptic operator in nonlinear potential theory. As an application of the Green function, we will establish interior Hölder continuity for degenerate subelliptic equations with inhomogeneous items.

Before stating our main results, we present several definitions which we will use in the next several sections:

Definition 2. Given any $f(x) \in\left[W^{1, p}(\Omega, X)\right]^{-1}$, which is the dual space of $W^{1, p}(\Omega, X)$, we say that $u \in W^{1, p}(\Omega, X)$ is a weak solution of equation $\mathcal{A} u=f(x)$ on $\Omega$ if and only if

$$
\mathcal{A}[u, \phi]:=\int_{\Omega}\langle A(x) \nabla u, \nabla u\rangle^{\frac{p-2}{2}}\langle A(x) \nabla u, \nabla \phi\rangle d x=\int_{\Omega} f(x) \phi(x) d x,
$$

for any $\phi(x) \in W_{0}^{1, p}(\Omega, X)$.

For each given $y \in \Omega$, we may rewrite the Green function of nonlinear degenerate equations $\mathcal{A} u=\delta(y)$ in the weak form.

Definition 3. A function $G_{y}(x): \Omega \times \Omega \mapsto \mathbb{R} \cup\{\infty\}$ is called a Green function if for any $y \in \Omega$ and small $R>0$, we have $G(\cdot, y) \in W^{1, p}\left(\Omega \backslash B_{R}(y), X\right) \cap W_{0}^{1, p-1}(\Omega, X)$ and

$$
\mathcal{A}\left[G_{y}, \phi\right]:=\int_{\Omega}\left\langle A(x) \nabla G_{y}(x), \nabla G_{y}(x)\right\rangle^{\frac{p-2}{2}}\left\langle A(x) \nabla G_{y}(x), \nabla \phi(x)\right\rangle d x=\phi(y),
$$

for any $\phi(x) \in W_{0}^{1, p}(\Omega, X)$.

For our purpose, we still need to consider the following approximation about the Green function $G^{r}(x, y)$ for the degenerate elliptic operator $\mathcal{A}[G, \phi]$.

Definition 4. For each given $y \in \Omega$ and small $r>0$ such that $B_{r}(y) \subset \Omega$, a function $G_{y}^{r} \in W_{0}^{1, p}(\Omega)$ is called a modified Green function if it is a weak solution 
of the following equation:

$$
\mathcal{A} G_{y}^{r}=\delta^{r}(y),
$$

where $\delta^{r}(y)=\frac{\chi_{B_{r}}(y)}{\left|B_{r}(y)\right|}$, and $\chi_{B_{r}}(y)$ is a characteristic function on $B_{r}(y)[1,8,30,43]$.

On the basis of hypotheses (H2) and (H3), one can deduce that $\phi \mapsto f_{B_{r}} \phi(x) d x$ is a bounded linear functional on $W_{0}^{1, p}(\Omega, X)$ for any small $r>0$ and the test function $\phi \in W_{0}^{1, p}(\Omega, X)$ [4]. Then from (1.8) it follows that

$$
\int_{\Omega}\left\langle A(x) \nabla G_{y}^{r}(x), \nabla G_{y}^{r}(x)\right\rangle^{\frac{p-2}{2}}\left\langle A(x) \nabla G_{y}^{r}(x), \nabla \phi(x)\right\rangle d x=f_{B_{r}} \phi(x) d x,
$$

for any test function $\phi(x) \in W_{0}^{1, p}(\Omega)$.

Now, let us present the following maximum principle with inhomogeneous items.

Theorem 1. Let $f \in L^{q}(\Omega)$ and $Q / p<q<\infty$. There exists a positive constant $C$ which depends only on $\lambda, Q, p$ and $\Omega$, such that for every weak solution $u \in$ $W^{1, p}(\Omega, X)$ of $\mathcal{A} u=f$, we have the inequality

$$
\sup _{\Omega} u^{+} \leq \sup _{\partial \Omega} u^{+}+C\|f\|_{L^{q}(\Omega)}^{\frac{1}{p-1}}
$$

where $u^{+}=\max \{u, 0\}$ and we define

$$
\sup _{\partial \Omega} u^{+}=\inf \left\{l: u^{+} \leq l \text { on } \partial \Omega \text { in the trace sense of } W^{1, p}(\Omega, X)\right\} .
$$

For nonlinear degenerate operators with X-ellipticity, our main result of the Green function is stated as follows:

Theorem 2. Let $1<p<Q$. Suppose that $G(x, y) \in W_{0}^{1, p}(\Omega)$ is a Green function of nonlinear degenerate operators defined by (1.7) with the $X$-elliptic condition (1.2) under the structural conditions (H1)-(H3). Then we obtain that

(1) for any $B_{2 R}(y) \subset \Omega$, there exists a positive constant $C=C(\lambda, Q, p, \Omega, X)$ such that

$$
\int_{\Omega \backslash B_{R}}|X G(x, y)|^{p} d x \leq C R^{\frac{p-Q}{p-1}}
$$

(2) for any $x, y \in \Omega$ satisfying $B_{d(x, y)}(x) \subset \Omega$, there exists a constant $C_{1}=$ $C_{1}(\lambda, Q, p, \Omega, X)>0$ such that

$$
G(x, y) \leq C_{1} d(x, y)^{\frac{p}{p-1}}\left|B_{d(x, y)}(x)\right|^{-\frac{1}{p-1}} ;
$$

(3) for any $x, y \in \Omega(x \neq y)$ satisfying $d(x, y) \leq \frac{2}{3} \operatorname{dist}(y, \partial \Omega)$ and $B_{d(x, y)}(x) \subset \Omega$, there exists another constant $C_{2}>0$ such that

$$
G(x, y) \geq C_{2}(\lambda, Q, p, \Omega, X) d(x, y)^{\frac{p}{p-1}}\left|B_{d(x, y)}(x)\right|^{-\frac{1}{p-1}} .
$$

In view of both estimates (1.10) and (1.11), we obtain a local behavior of the Green function as follows:

$$
G(x, y) \simeq \frac{1}{d(x, y)^{\frac{Q-p}{p-1}}}, \quad \forall x, y \in B_{R} \subset \Omega .
$$

This is an important tool to investigate the regularity in Morrey's spaces of linear and nonlinear operators with subellipticity and the Wiener criterion of the corresponding regular boundary points. 
In the present paper, as an application of the Green function to the regularity in Morrey's spaces, we consider the following nonlinear degenerate subelliptic equation of divergence form:

$$
-\sum_{k=1}^{m} X^{*}\left\{\left\langle A(x) X_{k} u, X_{k} u\right\rangle^{\frac{p-2}{2}} A(x) X_{k} u\right\}=f(x)-\sum_{k=1}^{m} X_{k} g(x),
$$

where $f(x) \in L^{q}(\Omega)$ and $g(x) \in\left[L^{s}(\Omega)\right]^{N}$ with both $q$ and $s>1$. Assume that $u \in L^{p}(\Omega)$ with $p \geq 1$ and $\lambda \geq 0$. We will use a version of Morrey's spaces modelled on X-control distances, in which various Morrey regularities for quasilinear X-elliptic operators were discussed by Di Fazio and his collaborators [14, 15, etc., that is,

$$
L^{p, \lambda}(\Omega)=\left\{\left.u \in L^{p}(\Omega)\left|\sup _{\substack{x_{0} \in \Omega \\ 0<\rho \leq d}} \frac{1}{\rho^{\lambda}} \int_{B_{\rho}\left(x_{0}\right)}\right| u\right|^{p} d x<+\infty\right\} .
$$

Its norm is defined by

$$
\|u\|_{L^{p, \lambda}(\Omega)}=\sup _{\substack{x_{0} \in \Omega \\ 0<\rho \leq d}}\left\{\frac{1}{\rho^{\lambda}} \int_{B_{\rho}\left(x_{0}\right)}|u|^{p} d x\right\}^{\frac{1}{p}},
$$

where $B_{\rho}\left(x_{0}\right)=B\left(x_{0}, \rho\right) \subset \Omega$ for any $x_{0} \in \Omega$ denotes an X-control ball in $\mathbb{R}^{N}$ of radius $\rho$ centered at $x_{0}$, and $d$ is the diameter of $\Omega$ [14, 15, 17. Our result is described as follows:

Theorem 3. Let $1<p<Q$. Suppose that $u \in W_{l o c}^{1, p}(\Omega, X)$ is any weak solution of the degenerate equation (1.12) under the $X$-elliptic condition (1.2) with the hypotheses (H1)-(H3). If $f(x) \in L^{q}(\Omega)$ and $g(x) \in\left[L^{s}(\Omega)\right]^{N}$ for $q>\frac{Q}{p}$ and $s>\frac{Q}{p-1}$, then we have

$$
X u \in L_{l o c}^{p, \lambda}(\Omega),
$$

for every $0<\lambda \leq Q-p+p \vartheta$ with some $0<\vartheta<1$, which only depends on $Q, \lambda, p, q$ and $s$.

As a direct conclusion of Theorem 3, if we enhance the regularity of the given data: $f(x) \in L^{q}(\Omega)$ and $g(x) \in\left[L^{s}(\Omega)\right]^{N}$ for $q>\frac{Q}{p}$ and $s>\frac{Q}{p-1}$, by using Morrey's type lemma [4], we have locally Hölder continuity with control metric for the weak solution of equation (1.12) as follows.

Corollary 1. Let $u \in W^{1, p}(\Omega, X)(1<p<Q)$ be a weak solution of the degenerate subelliptic equation (1.12). If $f(x) \in L^{q}(\Omega)$ and $g(x) \in\left[L^{s}(\Omega)\right]^{N}$ for $q>\frac{Q}{p}$ and $s>\frac{Q}{p-1}$, then there exists a constant depending on $N, p, Q, \Omega,\|f(x)\|_{L^{q}}$ and $\|g(x)\|_{L^{s}}$ such that

$$
|u(x)-u(y)| \leq C d(x, y)^{\vartheta}, \quad \forall x, y \in \Omega^{\prime} \Subset \Omega,
$$

where $0<\vartheta<1$ is the same as given in Theorem 3.

The rest of this paper is organized as follows: in the next section, we will present a maximum principle for inhomogeneous degenerate equations with the X-elliptic condition. In Section 3, for the sake of convenience, we show some basic properties for homogeneous degenerate equations (the so-called $\mathcal{A}$-harmonic equations). Section 4 is devoted to main contributions of our article, namely, the local estimates 
and comparisons of the modified Green functions. We will use them as an important tool for an approximating sequence in the proof of Theorem 2. In Section 5, we demonstrate the proof of Theorem 2. In Section 6, an application of the Green function is given to illustrate the regularity for a class of nonlinear subelliptic problems. Section 7 presents a brief conclusion. Note that all proofs of our main results do not require the Hörmander condition.

\section{Maximum PRinCiPle With X-Ellipticity}

In this section, we assume that the operator $\mathcal{A}$ defined by (1.1) is uniformly $\mathrm{X}$-elliptic under condition (1.2) in $\Omega$, and $u$ is any weak solution under the distributional sense of (1.6). The purpose of this section is to apply and develop classical techniques, introduced in [1, 29, 26], to prove the maximum principle with inhomogeneous items, i.e., Theorem 1.

Proof of Theorem 1. We separate our proof into two parts.

Step 1. We first prove an inequality

$$
\sup _{\Omega} u^{+} \leq C\left(\|u\|_{L^{p}(\Omega)}+\|f\|_{L^{q}(\Omega)}^{\frac{1}{p-1}}\right),
$$

for every $u \in W^{1, p}(\Omega, X)$ such that when $u \leq 0$ on $\partial \Omega, u$ satisfies

$$
\mathcal{A}[u, v] \leq \int_{\Omega} f v d x
$$

for any $v \in W_{0}^{1, p}(\Omega, X)$, where $v \geq 0$ and $u v \geq 0$. Let $k>0$ be a constant that will be determined later. For $\beta>1$ and $M>k$, we define a function $H \in C^{1}[k,+\infty)$ by setting $H(z)=z^{\beta}-k^{\beta}$ for $z \in[k, M]$ and take $H$ to be linear with respect to $z$ for $z \geq M$. Let $w=u^{+}+k$ and

$$
\phi(x)=G(w(x))=\int_{k}^{w(x)}\left(H^{\prime}(s)\right)^{p} d s .
$$

Then $\phi \geq 0$ and $\phi u \geq 0$. Since $u \leq 0$ on $\partial \Omega$, it follows that $\phi \in W_{0}^{1, p}(\Omega, X)$. Moreover, substituting $\phi$ into (2.2), we have

$$
\mathcal{A}[u, \phi] \leq \int f \phi d x
$$

From the X-ellipticity of $\mathcal{A}$ we get

$$
\mathcal{A}[u, \phi] \geq \lambda^{\frac{p}{2}} \int_{\Omega}|X w|^{p} G^{\prime}(w) d x .
$$

Since $G(s) \leq s G^{\prime}(s)$ and $u \leq w$, we deduce

$$
\begin{aligned}
\lambda^{\frac{p}{2}} \int_{\Omega}|X w|^{p} G^{\prime}(w) d x & \leq \int_{\Omega}|f| w G^{\prime}(w) d x \\
& =\int_{\Omega} \frac{|f|}{w^{p-1}} w^{p} G^{\prime}(w) d x \leq \int_{\Omega} \frac{|f|}{k^{p-1}} w^{p} G^{\prime}(w) d x,
\end{aligned}
$$

that is,

$$
\int_{\Omega}|X(H(w))|^{p} d x \leq \int_{\Omega} \frac{|f|}{\lambda k^{p-1}}\left|w H^{\prime}(w)\right|^{p} d x .
$$


Since $u \leq 0$ on $\partial \Omega$ and $H(k)=0$, it follows that $H(w) \in W_{0}^{1, p}(\Omega, X)$. By Sobolev's inequality and Hölder's inequality, we deduce

$$
\begin{aligned}
\left(\int_{\Omega}|H(w)|^{\frac{Q p}{Q-p}} d x\right)^{\frac{Q-p}{Q}} & \leq C \int_{\Omega} \frac{|f|}{\lambda k^{p-1}}\left|w H^{\prime}(w)\right|^{p} d x \\
& \leq C\left(\int_{\Omega}\left(\frac{|f|}{k^{p-1}}\right)^{q} d x\right)^{\frac{1}{q}}\left(\int_{\Omega}\left|w H^{\prime}(w)\right|^{p q^{\prime}} d x\right)^{\frac{1}{q^{\prime}}},
\end{aligned}
$$

where $q^{\prime}=\frac{q-1}{q}$. Choosing $k=\|f\|_{L^{q}(\Omega)}^{\frac{1}{p-1}}$, we have

$$
\|H(w)\|_{L^{\frac{Q p}{Q-p}(\Omega)}} \leq C\left\|w H^{\prime}(w)\right\|_{L^{p q^{\prime}}(\Omega)},
$$

where $C=C(\lambda, Q, p, \Omega)$. Now letting $M \rightarrow \infty$ and $H^{\prime}(w)=\beta z^{\beta-1}$ for $z \leq M$, we get

$$
\left\|w^{\beta}-k^{\beta}\right\|_{L^{\frac{Q p}{Q-p}(\Omega)}} \leq C \beta\|w\|_{L^{\beta p q^{\prime}}(\Omega)}^{\beta} .
$$

Hence, we deduce

$$
\begin{aligned}
\left(\int_{\Omega} w^{\beta \frac{Q p}{Q-p}} d x\right)^{\frac{Q-p}{Q p}} & \leq\left(\int_{\Omega}\left|w^{\beta}-k^{\beta}\right|^{\frac{Q p}{Q-p}} d x\right)^{\frac{Q-p}{Q p}}+k^{\beta} \\
& \leq C \beta\left(\int_{\Omega} w^{\beta p q^{\prime}} d x\right)^{\frac{1}{p q^{\prime}}}+\frac{1}{|\Omega|}\left(\int_{\Omega} k^{\beta p q^{\prime}} d x\right)^{\frac{1}{p q^{\prime}}} \\
& \leq C \beta\|w\|_{L^{\beta p q^{\prime}}(\Omega)}^{\beta},
\end{aligned}
$$

that is,

$$
\|w\|_{L^{\beta \frac{Q p}{Q-p}(\Omega)}} \leq(C \beta)^{1 / \beta}\|w\|_{L^{\beta p q^{\prime}(\Omega)}},
$$

where $\beta>1$. If we let $\chi=\frac{Q p /(Q-p)}{p q^{\prime}}$, the above inequality yields

$$
\|w\|_{L^{\beta p q^{\prime} \chi(\Omega)}} \leq(C \beta)^{1 / \beta}\|w\|_{L^{\beta p q^{\prime}}(\Omega)} .
$$

Taking $\beta=\chi^{m}, m=0,1, \cdots$ in (2.4) leads to

$$
\|w\|_{L^{p q^{\prime}} \chi^{m+1}(\Omega)} \leq \prod_{j=0}^{m}\left(C \chi^{j}\right)^{\chi^{-j}}\|w\|_{L^{p q^{\prime}}(\Omega)} .
$$

Letting $m \rightarrow \infty$ in (2.5), we derive

$$
\sup _{\Omega} w \leq C\|w\|_{L^{p q^{\prime}}(\Omega)} .
$$

Applying the interpolation inequality gives

$$
\sup _{\Omega} w \leq C\|w\|_{L^{p}(\Omega)} .
$$

Since $w=u^{+}+k$, we obtain the estimate (2.1).

Step 2. Let $l=\sup _{\partial \Omega} u^{+}$and $M=\sup _{\Omega} u^{+}$. We may assume $l=0$ and let $k>0$ be a constant to be determined later. We choose the test function

$$
\phi=\frac{1}{\left(M+k-u^{+}\right)^{p-1}}-\frac{1}{(M+k)^{p-1}} \in W_{0}^{1, p}(\Omega, X)
$$

and

$$
X \phi=\frac{(p-1) X u^{+}}{\left(M+k-u^{+}\right)^{p}} .
$$


By substituting this into (2.2) and using (1.3), we have

$$
\frac{p-1}{\lambda^{\frac{p}{2}}} \int_{\Omega} \frac{\left|X u^{+}\right|^{p}}{\left(M+k-u^{+}\right)^{p}} \leq \int_{\Omega}|f||\phi| \leq \int_{\Omega} \frac{|f|}{\left(M+k-u^{+}\right)^{p-1}} \leq \int_{\Omega} \frac{|f|}{k^{p-1}} .
$$

If we pick $k=\|f\|_{L^{q}(\Omega)}^{\frac{1}{p-1}}$ and $w=\log \frac{M+k}{M+k-u^{+}}$, then we have

$$
\int_{\Omega}|X w|^{p} d x \leq C(\lambda, Q, p, \Omega) .
$$

By Sobolev's inequality we deduce

$$
\|w\|_{L^{p}(\Omega)} \leq C(\lambda, Q, p, \Omega) .
$$

The proof will be completed if we can show that $w$ satisfies

$$
\mathcal{A}[w, \eta] \leq \int_{\Omega} \frac{|f|}{k^{p-1}} \eta d x
$$

for all $\eta \in W_{0}^{1, p}(\Omega, X)$ such that $\eta \geq 0$ and $\eta u \geq 0$ in $\Omega$. In fact, due to

$$
\nabla w=\frac{\nabla u}{M+k-u^{+}}
$$

taking

then we have

$$
\phi=\frac{\eta}{\left(M+k-u^{+}\right)^{p-1}},
$$

$$
\nabla \phi=\frac{\nabla \eta}{\left(M+k-u^{+}\right)^{p-1}}+\frac{\eta}{\left(M+k-u^{+}\right)^{p}} \nabla u^{+} .
$$

Since $\eta \geq 0$ and $\eta u \geq 0$, we have $\eta \nabla u^{+}=\eta \nabla u$. So using $\phi$ as a test function into (2.2) yields

$$
\begin{aligned}
\int_{\Omega}\langle A & \left.(x) \nabla u^{+}, \nabla u^{+}\right\rangle^{\frac{p-2}{2}}\left\langle A(x) \nabla u^{+}, \frac{\nabla \eta}{\left(M+k-u^{+}\right)^{p-1}}\right\rangle d x \\
& +\int_{\Omega}\left\langle A(x) \nabla u^{+}, \nabla u^{+}\right\rangle^{\frac{p-2}{2}}\left\langle A(x) \nabla u^{+}, \frac{\eta}{\left(M+k-u^{+}\right)^{p}} \nabla u^{+}\right\rangle d x \\
& =\int_{\Omega} f(x) \cdot \frac{\eta}{\left(M+k-u^{+}\right)^{p-1}} d x .
\end{aligned}
$$

From (1.3) and (1.4), the second term on the left-hand side is nonnegative, so we can drop it to obtain (2.7). Using the estimate (2.1) to $w$, we get

$$
\sup _{\Omega} w \leq C\left(\|w\|_{L^{p}(\Omega)}+\left\|\frac{f}{k^{p-1}}\right\|_{L^{q}(\Omega)}^{\frac{1}{p-1}}\right),
$$

for $Q / 2<p<\infty$. By the choice of $k$ and inequality (2.6) we obtain

$$
\sup _{\Omega} w \leq C .
$$

Choosing $k$ such that $M \leq k\left(e^{C}+1\right)$, we obtain

$$
\sup _{\Omega} w \leq C\|f\|_{L^{q}(\Omega)}^{\frac{1}{p-1}}
$$

for $Q / 2<p<\infty$. Since $w=u^{+}+k$, combining Step 1 and Step 2, we have completed the proof of Theorem 1. 


\section{Weak Harnack's inequality for $\mathcal{A}$-Harmonic With X-Ellipticity}

Due analogously to the degenerate elliptic case [32, Chap. 3 and Chap. 4], the following existence, uniqueness and comparison principles of weak solutions of homogeneous equations follow by the theory of monotone operators or by variational integral arguments under the X-elliptic condition (1.2).

Theorem 4. Suppose that $\Omega$ is a bounded domain and that $v \in W^{1, p}(\Omega)$. Then there is a unique solution $u \in W^{1, p}(\Omega)$ of the equation $\mathcal{A} u=0$ in $\Omega$ with $u-v \in$ $W_{0}^{1, p}(\Omega)$.

Theorem 5. If $u$ is a solution to the equation $\mathcal{A} u=0$ in the distributional sense in the domain $\Omega$ with the boundary value $v$, then we have

$$
\underset{x \in \Omega}{\operatorname{ess} \inf } v \leq u \leq \underset{x \in \Omega}{\operatorname{ess} \sup } v, \text { almost everywhere in } \Omega .
$$

Some local estimates for solutions of the homogeneous degenerate elliptic equation $\mathcal{A} u=0$ under the X-elliptic condition (1.2) also follow analogously to the degenerate elliptic case [6, 7, 32, 47]. Inserting a test function $\psi=\eta^{p} u$ with a nonnegative cutoff function $\eta \in C_{0}^{1}(\Omega, X)$ into the equation of the weak form, then from the X-elliptic condition (1.2) we obtain a Caccioppoli type inequality:

$$
\int_{\Omega} \eta^{p}|X u|^{p} d x \leq C(\lambda, Q, p) \int_{\Omega}|X \eta|^{p}|u|^{p} .
$$

Furthermore, by using Moser's iteration technique the following locally upper boundedness of the weak solution for $\mathcal{A} u=0$ has been proved [32, Theorem 3.59]. We list those results below which will be used in the subsequent arguments: there are positive constants $C_{1}$ and $C_{2}$ which depend on $\lambda, Q, p, \Omega$ and $X$ such that

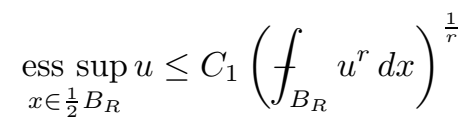

and

$$
\underset{x \in B_{R}}{\operatorname{ess} \inf _{R}} u \geq C_{2}\left(f_{B_{R}} u^{-r} d x\right)^{-\frac{1}{r}},
$$

for any $r>0$, where $B_{R}$ is a ball with $2 B_{R} \subset \Omega$.

Based on the results on the spaces of bounded mean oscillation functions introduced by John and Nirenberg [29, the following weak Harnack's inequality is valid for the equation $\mathcal{A} u=0$ [4].

Theorem 6 (Weak Harnack's inequality). Let $u \in W^{1, p}(\Omega, X)$ be a nonnegative weak supersolution of the homogeneous equation $\mathcal{A}[u, \phi]=0$ with the $X$-elliptic assumption (1.2). Then, for $B_{R}(x) \subset \Omega, r<\frac{Q(p-1)}{Q-p}$ if $p<Q, R<R_{0}$ and $0<\sigma<\tau<1$, there exists a positive constant $C=C(\lambda, Q, p, \sigma, \tau, \Omega, X)$ such that

$$
\left(f_{B_{\tau R}} u^{r} d x\right)^{\frac{1}{r}} \leq C \underset{B_{\sigma R}}{\operatorname{ess} \inf } u
$$

where $R_{0}$ depends on $X$ and $\Omega$.

By means of the weak Harnack's inequality and the classical procedure described in [32, 47, we have the following local Hölder estimates for weak solutions of the homogeneous equation $\mathcal{A}[u, \phi]=0$ with respect to both Carnot-Carathéodory and 
Euclidean metrics. Namely, for any subdomain $\Omega^{\prime} \subset \Omega$, there exist constants $C$ and $\alpha>0$ dependent of $X, \Omega^{\prime}, \Omega$ and $\lambda$ such that

$$
\sup _{x, y \in \Omega^{\prime}} \frac{|u(x)-u(y)|}{d^{\alpha}(x, y)} \leq C \sup _{\Omega}|u|
$$

and

$$
\sup _{x, y \in \Omega^{\prime}} \frac{|u(x)-u(y)|}{|x-y|^{\alpha / r}} \leq C \sup _{\Omega}|u|,
$$

where $r$ is the Hörmander rank with respect to $X$.

We also have a full Harnack's inequality for nonnegative weak solutions of the homogeneous equation $\mathcal{A}[u, \phi]=0$. Namely, for $B_{R}(x) \subset \Omega, R<R_{0}$ and $0<\tau<1$, we have

$$
\sup _{x \in B_{\tau R}} u \leq C \inf _{x \in B_{\tau R}} u,
$$

where $R_{0}$ is the same as given in Theorem 6 and $C>0$ is a constant which depends on $Q, p, \Omega, X, \tau$ and $\lambda$. Moreover, the above Harnack's inequality also holds in shells $B_{\tau R}-\bar{B}_{\sigma R}$ for $0<\sigma<\tau<1$ [6, 7].

\section{Estimates of MOdified GReEn FUnCtions}

In this section, we will present some estimates involving their norms in the weak$L^{p}$ space. Let us recall the definition of the weak- $L^{p}$ space. For $f \in L^{p}(\Omega)$ and $t>0$, let $\Omega_{t}(f):=\{x \in \Omega|| f(x) \mid>t\}$ and $\lambda_{f}(t):=\left|\Omega_{t}(f)\right|$. Then the weak- $L^{p}$ space is denoted by $L_{w}^{p}(\Omega)$, which satisfies

$$
\|f\|_{L_{w}^{p}(\Omega)}:=\inf \left\{B \mid \lambda_{f}(t) \leq t^{-p} B^{p}, \forall t>0\right\}<\infty,
$$

for all $p \geq 1$. In the proofs of our results, we will need the following two inequalities concerning $\|f\|_{L_{w}^{p}(\Omega)}$ :

$$
\|f\|_{L_{w}^{p}(\Omega)} \leq\|f\|_{L^{p}(\Omega)}, \quad p>1,
$$

and

$$
\left|f\left\|_{L^{q}(\Omega)} \leq\left(\frac{q}{p-q}\right)^{\frac{1}{q}}|\Omega|^{\frac{1}{q}-\frac{1}{p}}\right\| f \|_{L_{w}^{p}(\Omega)}, \quad 1 \leq q<p .\right.
$$

Now we are in a position to prove several important conclusions about the modified Green functions due to equation (1.9).

Lemma 1. Suppose that $G_{y}^{r}(x)$ is a weak solution of equation (1.9) with the $X$ elliptic condition (1.2) in the sense of distributions. Then $G_{y}^{r}(x) \geq 0$, almost everywhere in $\Omega$.

Proof of Lemma 1. For the fixed $y \in \Omega$, let $r>0$ be small enough such that $\bar{B}_{r}(y) \subset \Omega$. Taking $G_{y}^{r}$ and $\left|G_{y}^{r}\right| \in W_{0}^{1, p}(\Omega, X)$ as the test functions, we have

$$
0 \leq \mathcal{A}\left[G_{y}^{r}, G_{y}^{r}\right]=f_{B_{r}(y)} G_{y}^{r} d x \leq f_{B_{r}(y)}\left|G_{y}^{r}\right| d x=\mathcal{A}\left[G_{y}^{r},\left|G_{y}^{r}\right|\right],
$$

which yields

$$
\mathcal{A}\left[G_{y}^{r}, G_{y}^{r}-\left|G_{y}^{r}\right|\right] \leq 0
$$


Let $\Omega^{+}=\left\{x \in \Omega \mid G_{y}^{r} \geq 0\right\}$ and $\Omega^{-}=\left\{x \in \Omega \mid G_{y}^{r}<0\right\}$. Taking the X-elliptic condition (1.2) into account, we have

$$
\begin{aligned}
0 & \geq \int_{\Omega}\left\langle A(x) \nabla G_{y}^{r}, \nabla G_{y}^{r}\right\rangle^{\frac{p-2}{2}}\left\langle A(x) \nabla G_{y}^{r}, \nabla\left(G_{y}^{r}-\left|G_{y}^{r}\right|\right)\right\rangle d x \\
& =2 \int_{\Omega^{-}}\left\langle A(x) \nabla G_{y}^{r}, \nabla G_{y}^{r}\right\rangle^{\frac{p-2}{2}}\left\langle A(x) \nabla G_{y}^{r}, \nabla G_{y}^{r}\right\rangle d x \geq \frac{2}{\lambda^{\frac{p}{2}}} \int_{\Omega^{-}}\left|X G_{y}^{r}\right|^{p} d x .
\end{aligned}
$$

So we deduce

$$
\left|\Omega^{-}\right|=0
$$

that is,

$$
G_{y}^{r} \geq 0, \quad \text { almost everywhere in } \Omega \text {. }
$$

This completes the proof of Lemma 1.

Theorem 7. For $1<p<Q$, suppose that $G_{y}^{r}(x)$ is a weak solution of equation (1.9) in the sense of distributions for small $r>0$ and the fixed point $y \in \Omega$. Then the modified Green function $G_{y}^{r}(x)$ enjoys the following property:

$$
G_{y}^{r} \in L_{w}^{\kappa}(\Omega) \quad \text { for } \quad \kappa=\frac{(p-1) Q}{Q-p},
$$

with

$$
\left\|G^{r}(\cdot, y)\right\|_{L_{w}^{\kappa}(\Omega)}<C(\lambda, Q, p, \Omega, X) .
$$

Proof of Theorem 7. For any $t>0$, we set $\Omega_{t}:=\left\{x \in \Omega|| G_{y}^{r} \mid>t\right\}$ and consider the function $f(s)=\left[\frac{1}{t^{p-1}}-\frac{1}{s^{p-1}}\right]^{+}:=\max \left\{0, \frac{1}{t^{p-1}}-\frac{1}{s^{p-1}}\right\} \in \operatorname{Lip}(\mathbb{R})$. Since $G_{y}^{r}(x) \in W_{0}^{1, p}(\Omega, X)$, from [26, 43] we have $f\left[G_{y}^{r}(x)\right] \in W_{0}^{1, p}(\Omega, X)$ and $f\left[G_{y}^{r}(x)\right]^{+} \in$ $W_{0}^{1, p}(\Omega, X)$. We pick $\phi(x):=f\left[G_{y}^{r}(x)\right]^{+}$as a test function. By considering that $f(s)<\frac{1}{t^{p-1}}$ for any $s \in \mathbb{R}$, we get

$$
\begin{aligned}
\mathcal{A}\left[G_{y}^{r}, \phi\right] & =\int_{\Omega}\left\langle A(x) \nabla G_{y}^{r}, \nabla G_{y}^{r}\right\rangle^{\frac{p-2}{2}}\left\langle A(x) \nabla G_{y}^{r}, \nabla \phi(x)\right\rangle d x \\
& =f_{\Omega} \phi(x) d x \\
& =f_{\Omega} \frac{1}{t^{p-1}} d x \leq \frac{1}{t^{p-1}} .
\end{aligned}
$$

On the other hand, by virtue of (1.3) and (1.4) we deduce

$$
\begin{aligned}
\mathcal{A}\left[G_{y}^{r}, \phi\right] & =\int_{\Omega}\left\langle A(x) \nabla G_{y}^{r}, \nabla G_{y}^{r}\right\rangle^{\frac{p-2}{2}}\left\langle A(x) \nabla G_{y}^{r}, \nabla \phi(x)\right\rangle d x \\
& =\int_{\Omega}\left\langle A(x) \nabla G_{y}^{r}, \nabla G_{y}^{r}\right\rangle^{\frac{p-2}{2}}\left\langle A(x) \nabla G_{y}^{r},(p-1) \frac{\nabla G_{y}^{r}}{\left(G_{y}^{r}\right)^{p}}\right\rangle d x \\
& \geq\left(\frac{1}{\lambda^{\frac{p-2}{2}}}\right)\left|X G_{y}^{r}\right|^{p-2} \int_{\Omega_{t}}\left\langle A(x) \nabla G_{y}^{r},(p-1) \frac{\nabla G_{y}^{r}}{\left(G_{y}^{r}\right)^{p}}\right\rangle d x \\
& =(p-1)\left(\frac{1}{\lambda^{\frac{p-2}{2}}}\right)\left|X G_{y}^{r}\right|^{p-2} \int_{\Omega_{t}} \frac{1}{\left(G_{y}^{r}\right)^{p}}\left\langle A(x) \nabla G_{y}^{r}, \nabla G_{y}^{r}\right\rangle d x \\
& \geq \frac{p-1}{\lambda^{\frac{p}{2}}} \int_{\Omega_{t}}\left|X G_{y}^{r}\right|^{p}\left(G_{y}^{r}\right)^{-p} d x \\
& =\frac{p-1}{\lambda^{\frac{p}{2}}} \int_{\Omega_{t}}\left|X \log G_{y}^{r}\right|^{p} d x .
\end{aligned}
$$


Combining (4.3) and (4.4) yields

$$
\int_{\Omega_{t}}\left|X \log G_{y}^{r}\right|^{p} d x \leq \frac{\lambda^{\frac{p}{2}}}{(p-1) t^{p-1}} .
$$

By Sobolev's imbedding inequality it follows that

$$
\begin{aligned}
\left(\int_{\Omega_{t}}\left|\log \frac{G_{y}^{r}}{t}\right|^{\frac{Q p}{Q-p}} d x\right)^{\frac{Q-p}{Q}} & =\left(\int_{\Omega_{t}}\left|\log G_{y}^{r}-\log t\right|^{\frac{Q p}{Q-p}} d x\right)^{\frac{Q-p}{Q}} \\
& \leq C \int_{\Omega_{t}}\left|X \log G_{y}^{r}\right|^{p} d x \\
& \leq \frac{C \lambda^{\frac{p}{2}}}{(p-1) t^{p-1}} .
\end{aligned}
$$

Now let us consider $\Omega_{2 t}$. Apparently, one can see that $\Omega_{2 t} \subset \Omega_{t}$ and $(\log 2)^{\frac{Q p}{Q-p}}<$ $\left(\log G_{y}^{r} / t\right)^{\frac{Q p}{Q-p}}$ when $\frac{G_{y}^{r}}{t}>2$ on $\Omega_{2 t}$. So we have

$$
\begin{aligned}
(\log 2)^{p}\left|\Omega_{2 t}\right|^{\frac{Q-p}{Q}} & =\left(\int_{\Omega_{2 t}}(\log 2)^{\frac{Q p}{Q-p}} d x\right)^{\frac{Q-p}{Q}} \\
& \leq\left(\int_{\Omega_{2 t}}\left(\log \frac{G_{y}^{r}}{t}\right)^{\frac{Q p}{Q-p}} d x\right)^{\frac{Q-p}{Q}} \\
& \leq\left(\int_{\Omega_{t}}\left(\log \frac{G_{y}^{r}}{t}\right)^{\frac{Q p}{Q-p}} d x\right)^{\frac{Q-p}{Q}} \\
& \leq \frac{C \lambda^{\frac{p}{2}}}{(p-1) t^{p-1}},
\end{aligned}
$$

which implies that

$$
2 t^{p-1}\left|\Omega_{2 t}\right|^{\frac{Q-p}{Q}} \leq C(\lambda, Q, p, \Omega, X) .
$$

On the basis of the definition of the weak- $L^{p}$ space we obtain

$$
G_{y}^{h}(x) \in L_{w}^{\kappa}(\Omega), \quad \kappa=\frac{(p-1) Q}{Q-p} .
$$

Consequently, this completes the proof of Theorem 7 .

Theorem 8. Suppose that $G_{y}^{r} \in W_{0}^{1, p}(\Omega, X)$ is a modified Green function of the approximating $\mathcal{A}$-harmonic equation (1.9) under the $X$-elliptic assumption (1.2). Then there exists a constant $C=C(\lambda, Q, p, \Omega, X)>0$ such that

$$
G_{y}^{r}(x) \leq C r^{\frac{p}{p-1}}\left|B_{r}(y)\right|^{-\frac{1}{p-1}}, \quad \text { if } \quad d(x, y) \leq 2 r,
$$

and

$$
G_{y}^{r}(x) \leq C d(x, y)^{\frac{p}{p-1}}\left|B_{d(x, y)}(x)\right|^{-\frac{1}{p-1}}, \quad \text { if } \quad d(x, y) \geq 2 r .
$$

Proof of Theorem 8. Let us divide our proof into two cases.

Case 1. For given $y \in \Omega$, let $x \in \Omega$ satisfy $d(x, y) \leq 2 r$. As the modified Green function $G_{y}^{r} \in W_{0}^{1, p}(\Omega, X)$ is a nonnegative weak solution of equation $\mathcal{A} u=\frac{\chi_{B_{r}(y)}}{\left|B_{r}(y)\right|}$, 
from the maximum principle (Theorem 1) we get

$$
G_{y}^{r} \leq \sup _{\Omega} G_{y}^{r} \leq C\left\|\frac{\chi_{B_{r}(y)}}{\left|B_{r}(y)\right|}\right\|_{L^{q}(\Omega)}^{\frac{1}{p-1}}=C\left|B_{r}(y)\right|^{\frac{1}{(p-1) q}-\frac{1}{p-1}},
$$

which holds for every $q>\frac{Q}{p}$. Let us remark that $C$ is independent of $G_{y}^{r}, y$ and $r$. Letting $q \rightarrow \frac{Q}{p}$ we get

$$
G_{y}^{r}(x) \leq C\left|B_{r}(y)\right|^{\frac{p}{(p-1) Q}-\frac{1}{p-1}} .
$$

When $r$ gets sufficiently small, we can use the assumption (H2) to derive

$$
G_{y}^{r}(x) \leq \operatorname{Cr}^{\frac{p}{p-1}}\left|B_{r}(y)\right|^{-\frac{1}{p-1}},
$$

where $C$ is independent of $r$ and $y \in \Omega$.

Case 2. Choose $x_{0} \in \Omega$ such that $d\left(x_{0}, y\right) \geq 2 r$ and set $R:=d\left(x_{0}, y\right)$. There are two subcases in our discussions.

Subcase 2.1. When $B_{R / 2}\left(x_{0}\right) \subset \Omega$, the modified Green function $G_{y}^{r}$ is a nonnegative weak solution of equation $\mathcal{A} u=0$ on $\Omega \backslash B_{r}(y)$ and $B_{R / 2}\left(x_{0}\right) \subset \Omega \backslash B_{r}(y)$. Then, according to formula (2.3), when $q \geq 1$, for every $x \in B_{R / 2}\left(x_{0}\right)$, we have

$$
G_{y}^{r}(x) \leq C\left(f_{B_{R / 2}\left(x_{0}\right)}\left(G_{y}^{r}\right)^{q} d x\right)^{\frac{1}{q}},
$$

where $C=C(\lambda, Q, p, q, \Omega, X)$. Let $B=B_{R / 2}\left(x_{0}\right)$ and $q \in[1, \kappa)$. In view of inequality (4.1) we have

$$
\begin{aligned}
\left\|G_{y}^{r}\right\|_{L^{q}(B)} & \leq\left(\frac{\kappa}{\kappa-q}\right)^{\frac{1}{q}}|B|^{\frac{1}{q}-\frac{1}{\kappa}}\left\|G_{y}^{r}\right\|_{L_{w}^{\kappa}(B)} \\
& \leq\left(\frac{\kappa}{\kappa-q}\right)^{\frac{1}{q}}|B|^{\frac{1}{q}-\frac{1}{\kappa}}\left\|G_{y}^{r}\right\|_{L_{w}^{\kappa}(\Omega)},
\end{aligned}
$$

that is,

$$
\left(f_{B}\left(G_{y}^{r}\right)^{q} d x\right)^{\frac{1}{q}} \leq\left(\frac{\kappa}{\kappa-q}\right)^{\frac{1}{q}}|B|^{-\frac{1}{\kappa}}\left\|G_{y}^{r}\right\|_{L_{w}^{\kappa}(\Omega)} .
$$

Due to $\left\|G_{y}^{r}\right\|_{L_{w}^{\kappa}(\Omega)} \leq C(\lambda, Q, p, \Omega, X)$, we have

$$
\left(f_{B}\left(G_{y}^{r}\right)^{q} d x\right)^{\frac{1}{q}} \leq C|B|^{-\frac{1}{\kappa}} .
$$

By using (4.6) we deduce

$$
\begin{aligned}
G_{y}^{r} & \leq C\left|B_{R / 2}\left(x_{0}\right)\right|^{-\frac{1}{\kappa}} \leq C\left|B_{R}\left(x_{0}\right)\right|^{-\frac{1}{\kappa}} \\
& =C\left|B_{d\left(x_{0}, y\right)}\left(x_{0}\right)\right|^{\frac{p}{(p-1) Q}}\left|B_{d\left(x_{0}, y\right)}\left(x_{0}\right)\right|^{-\frac{1}{p-1}} .
\end{aligned}
$$

If $R=d\left(x_{0}, y\right) \leq R_{0}$, from the assumption (H2) we immediately find

$$
\left|B_{d\left(x_{0}, y\right)}\left(x_{0}\right)\right|^{\frac{p}{(p-1) Q}} \leq C d\left(x_{0}, y\right)^{\frac{p}{p-1}} .
$$

On the contrary, if $R=d\left(x_{0}, y\right)>R_{0}$, we choose $t=R / R_{0}>1$. On the basis of assumption (H2) and (1.5), we deduce

$$
\begin{aligned}
\left|B_{d\left(x_{0}, y\right)}\left(x_{0}\right)\right|^{\frac{p}{(p-1) Q}} & \leq A^{\frac{p}{(p-1) Q}} t^{\frac{p}{p-1}}\left|B_{R_{0}}\left(x_{0}\right)\right|^{\frac{p}{(p-1) Q}} \\
& \leq A^{\frac{p}{(p-1) Q}} t^{\frac{p}{p-1}} C R_{0}^{\frac{p}{p-1}} \leq C d\left(x_{0}, y\right)^{\frac{p}{p-1}} .
\end{aligned}
$$


So in either case for (4.7), we obtain

$$
G_{y}^{r}(x) \leq C d\left(x_{0}, y\right)^{\frac{p}{p-1}}\left|B_{d\left(x_{0}, y\right)}\left(x_{0}\right)\right|^{-\frac{1}{p-1}},
$$

for every $x \in B_{R / 2}\left(x_{0},\right)$. Taking $x=x_{0}$, we obtain (4.5) with $C$ being independent of $r$.

Subcase 2.2. When $B_{R / 2}\left(x_{0}\right) \subset \mathbb{R}^{N} \backslash \Omega$, let $\tilde{\Omega}$ be an open bounded set such that $B_{R / 2}\left(x_{0}\right) \cup \Omega \subset \tilde{\Omega}$. It is possible to extend the operator $\mathcal{A}$ to $\tilde{\Omega}$, so let $\tilde{G}_{y}^{r}$ be the corresponding modified Green function in $\tilde{\Omega}$. By using a similar argument as shown in Case 1, the following inequality holds:

$$
\tilde{G}_{y}^{r} \leq C d\left(x_{0}, y\right)^{\frac{p}{p-1}}\left|B_{d\left(x_{0}, y\right)}\left(x_{0}\right)\right|^{-\frac{1}{p-1}} .
$$

So the result will immediately follow once we prove that $G_{y}^{r} \leq \tilde{G}_{y}^{r}$ on $\Omega$. Note that the function $\tilde{G}_{y}^{r}-G_{y}^{r}$ is a weak solution of equation $\mathcal{A} u=0$ due to the fact that $\mathcal{A} \tilde{G}_{y}^{r}=\mathcal{A} G_{y}^{r}$. From Lemma 1 we know that $\tilde{G}_{y}^{r} \geq 0$ on $\tilde{\Omega} \supset \Omega$; consequently, $-\tilde{G}_{y}^{r} \leq 0$ on $\Omega$. By using Theorem 6 it follows that $-\tilde{G}_{y}^{r} \leq 0$ on $\partial \Omega$, so we get

$$
G_{y}^{r}+\left(-\tilde{G}_{y}^{r}\right) \leq 0, \quad \text { on } \quad \partial \Omega .
$$

By virtue of Theorem 5, it follows that $\tilde{G}_{y}^{r} \geq G_{y}^{r}$ on $\Omega$. Therefore, the proof of Theorem 8 is completed.

Before proving our next two theorems, here we introduce another technical lemma:

Lemma 2. Suppose that $G_{y}^{r} \in W_{0}^{1, p}(\Omega, X)$ is a weak solution of the approximating $\mathcal{A}$-harmonic equation (1.9) under the X-elliptic assumption (1.2). Then we obtain

$$
\int_{\Omega \backslash B_{R}}\left|X G_{y}^{r}\right|^{p} d x \leq C(Q, p, \lambda, \Omega, X) R^{\frac{p-Q}{p-1}},
$$

for any $B_{R} \subset \Omega$.

Proof of Lemma 2. Let us fix $y \in \Omega$ for any small $r>0$.

Case 1. If $0<R<4 r$, using $\phi(x)=G_{y}^{r}(x)$ as a test function in $\mathcal{A}\left(G_{y}^{r}, \phi\right):=$ $f_{B_{r}} \phi(x) d x$, from Hölder's inequality and Sobolev's inequality we have

$$
\begin{aligned}
\mathcal{A}\left[G_{y}^{r}, G_{y}^{r}\right]=f_{B_{r}(y)} G_{y}^{r} d x & \leq\left|B_{r}(y)\right|^{\frac{p-Q}{Q p}}\left(\int_{B_{r}(y)}\left(G_{y}^{r}\right)^{\frac{Q p}{Q-p}}\right)^{\frac{Q-p}{Q p}} \\
& \leq C\left|B_{r}(y)\right|^{\frac{1}{Q}-\frac{1}{p}}\left(\int_{\Omega}\left|X G_{y}^{r}\right|^{p} d x\right)^{\frac{1}{p}}
\end{aligned}
$$

In view of the X-ellipticity assumption (1.2) we get

$$
\int_{\Omega}\left|X G_{y}^{r}\right|^{p} d x \leq C(\lambda, Q, p, \Omega, X)\left|B_{r}(y)\right|^{\frac{p}{(p-1) Q}-\frac{1}{p-1}} .
$$

Due to the assumption $r<R_{0}$, it is easy to see that $R / 4<r \leq R_{0}$. By virtue of (H2) we can find

$$
\left|B_{r}(y)\right|^{\frac{p}{(p-1) Q}-\frac{1}{p-1}} \leq C\left|B_{R / 4}(y)\right|^{\frac{p}{(p-1) Q}-\frac{1}{p-1}} \leq C R^{\frac{p-Q}{p-1}} .
$$


Using this inequality with (4.9) we deduce

$$
\int_{\Omega}\left|X G_{y}^{r}\right|^{p} d x \leq C(\lambda, Q, p, \Omega, X) R^{\frac{p-Q}{p-1}}
$$

Case 2. If $R \geq 4 r$, we pick a cutoff function $\eta \in C_{0}^{\infty}\left(B_{R}, X\right)$ such that $0 \leq$ $\eta \leq 1, \eta=1$ in $B_{R / 2}(y)$ and $\eta=0$ outside $B_{R}(y)$ as well as $|X \eta| \leq \frac{C}{R}$. Using $\phi(x)=(1-\eta)^{p} G_{y}^{r} \in W_{0}^{1, p}(\Omega, X)$ as a test function in (1.9), we have

$$
\mathcal{A}\left[G_{y}^{r},(1-\eta)^{p} G_{y}^{r}\right]=f_{B_{r}(y)}(1-\eta)^{p} G_{y}^{r} d x=0,
$$

where we have taken into account that it is $\eta=1 \mathrm{in} B_{r}(y)$. So we get

$$
\begin{aligned}
& \int_{\Omega}\left\langle A(x) \nabla G_{y}^{r}, \nabla G_{y}^{r}\right\rangle^{\frac{p}{2}}(1-\eta)^{p} d x \\
& \quad=-p \int_{\Omega}\left\langle A(x) \nabla G_{y}^{r}, \nabla G_{y}^{r}\right\rangle^{\frac{p-2}{2}}\left\langle A(x) \nabla G_{y}^{r}, \nabla(1-\eta)\right\rangle G_{y}^{r}(1-\eta)^{p-1} d x .
\end{aligned}
$$

Utilizing the X-ellipticity assumption (1.2) and Young's inequality we get

$$
\begin{aligned}
& \frac{1}{\lambda^{\frac{p}{2}}} \int_{\Omega}\left|X G_{y}^{r}\right|^{p}(1-\eta)^{p} d x \leq p \lambda^{\frac{p}{2}} \int_{\Omega}\left|X G_{y}^{r}\right|^{p-1}|X(1-\eta)|\left|G_{y}^{r}\right|(1-\eta)^{p-1} d x \\
& \leq \lambda^{\frac{p}{2}}\left(\frac{p-1}{p \lambda^{p}} \int_{\Omega}\left|X G_{y}^{r}\right|^{p}(1-\eta)^{p} d x+\left.\frac{1}{p \lambda^{p}} \int_{\Omega}|X(1-\eta)|^{p}|| G_{y}^{r}\right|^{p} d x\right),
\end{aligned}
$$

which implies

$$
\int_{\Omega}\left|X G_{y}^{r}\right|^{p}(1-\eta)^{p} d x \leq\left. C(\lambda, p) \int_{\Omega}|X(1-\eta)|^{p}|| G_{y}^{r}\right|^{p} d x
$$

From this inequality, since $\eta=1$ in $B_{R / 2}(y), \eta=0$ outside $B_{R}(y)$ and $|X \eta| \leq \frac{C}{R}$, it follows that

$$
\int_{\Omega \backslash B_{R}(y)}\left|X G_{y}^{r}\right|^{p} d x \leq \frac{C}{R^{p}} \int_{B_{R}(y) \backslash B_{R / 2}(y)}\left(G_{y}^{r}\right)^{p} d x .
$$

If $x \in B_{R}(y) \backslash B_{R / 2}(y)$, then we have $R>d(x, y) \geq R / 2 \geq 2 r$. Thus, we can use inequality (4.5), which holds provided that $r$ is sufficiently small, with $C$ being independent of $r$. In this case we need to consider the following two possibilities.

Subcase 2.1. If $d(x, y) \leq R_{0}$, then $R / 2 \leq d(x, y)<R$. So using (H2) we derive

$$
\begin{aligned}
d(x, y)^{\frac{p}{p-1}}\left|B_{d(x, y)}(x)\right|^{-\frac{1}{p-1}} & \leq R^{\frac{p}{p-1}} C_{1}^{-1} d(x, y)^{-\frac{Q}{p-1}} \\
& \leq R^{\frac{p}{p-1}} C_{2} C_{1}^{-1}\left|B_{d(x, y)}(y)\right|^{-\frac{1}{p-1}} \\
& \leq C R^{\frac{p}{p-1}}\left|B_{R / 2}(y)\right|^{-\frac{1}{p-1}} .
\end{aligned}
$$

Substituting the above inequality and (4.5) into (4.10) we get

$$
\begin{aligned}
\int_{\Omega \backslash B_{R}(y)}\left|X G_{y}^{r}\right|^{p} d x & \leq C R^{\frac{p}{p-1}}\left|B_{R / 2}(y)\right|^{-\frac{p}{p-1}}\left|B_{R}(y) \backslash B_{R / 2}(y)\right| \\
& \leq C R^{\frac{p}{p-1}}\left|B_{R / 2}(y)\right|^{-\frac{p}{p-1}}\left|B_{R}(y)\right| .
\end{aligned}
$$

From the assumption (H2), it follows that $\left|B_{R}(y)\right| \leq C R^{Q}$ for all $R>0$. From (H2) again it follows that $\left|B_{R / 2}(y)\right|^{-\frac{p}{p-1}} \leq C R^{-\frac{p Q}{p-1}}$. Then we have

$$
\int_{\Omega \backslash B_{R}(y)}\left|X G_{y}^{r}\right|^{p} d x \leq C R^{\frac{p-Q}{p-1}} .
$$


Subcase 2.2. If $d(x, y)>R_{0}$, it satisfies $\frac{R}{2} \leq d(x, y)<R$. By using (H2) we derive

$$
\begin{aligned}
d(x, y)^{\frac{p}{p-1}}\left|B_{d(x, y)}(x)\right|^{-\frac{1}{p-1}} & \leq d(x, y)^{\frac{p}{p-1}}\left|B_{R_{0}}(x)\right|^{-\frac{1}{p-1}} \\
& \leq d(x, y)^{\frac{p}{p-1}} C_{1}^{-1}\left|R_{0}\right|^{-\frac{Q}{p-1}} \\
& =C d(x, y)^{\frac{p}{p-1}} \leq C R^{\frac{p}{p-1}} .
\end{aligned}
$$

Combining (4.10) and (4.5) yields

$$
\int_{\Omega \backslash B_{R}(y)}\left|X G_{y}^{r}\right|^{p} d x \leq C R^{\frac{p}{p-1}}\left|B_{R}(y) \backslash B_{R / 2}(y)\right| \leq C R^{\frac{p}{p-1}}\left|B_{R}(y)\right| .
$$

Again, using (H2) we get $\left|B_{R}(y)\right| \leq C R^{Q}$ for all $R>0$. Moreover, given that $\Omega$ is bounded, there exists a constant $R_{1}>0$, which depends on $\lambda, Q, p, \Omega$ and $X$, such that $\Omega \subset B_{R_{1}}(y)$. If $R \leq R_{1}$, we have

$$
\begin{aligned}
\int_{\Omega \backslash B_{R}(y)}\left|X G_{y}^{r}\right|^{p} d x & \leq C R^{\frac{p}{p-1}}\left|B_{R}(y)\right| \\
& \leq C R^{\frac{p}{p-1}} R^{Q} \leq C R^{\frac{p}{p-1}} R^{Q}\left(\frac{R_{1}}{R}\right)^{\frac{p Q}{p-1}} \\
& =C(\lambda, Q, p, \Omega, X) R^{\frac{p-Q}{p-1}},
\end{aligned}
$$

which implies (4.8). On the contrary, if $R>R_{1}$, we have $\Omega \backslash B_{R}(y)=\emptyset$. So the proof of Lemma 2 is completed.

Theorem 9. Let $1<p<Q$. Suppose that $G_{y}^{r}(x)$ is any weak solution of the approximating $\mathcal{A}$-harmonic equation (1.9) under the X-elliptic assumption (1.2). Then

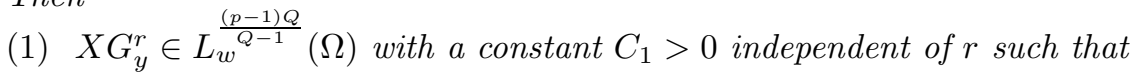

$$
\left\|X G_{y}^{r}\right\|_{L_{w}^{\frac{Q(p-1)}{Q-1}}(\Omega)} \leq C_{1}(\lambda, Q, p, \Omega, X) ;
$$

(2) $G^{r}(\cdot, y) \in W_{0}^{1, \nu}(\Omega, X)$ for $1 \leq \nu<\frac{(p-1) Q}{Q-1}$ with a constant $C_{2}>0$ independent of $r$ such that

$$
\left\|X G_{y}^{r}\right\|_{W^{1, \nu}(\Omega, X)} \leq C_{2}(\lambda, Q, p, \Omega, X) .
$$

Proof of Theorem 9. (1) Let $t>0, R=t^{-\frac{p-1}{Q-1}}$ and $\Omega_{t}=\left\{x \in \Omega:\left|X G_{y}^{r}\right|>t\right\}$. Obviously, $\Omega_{t} \subset \Omega$. Then we can write

$$
\begin{aligned}
\left|\Omega_{t}\right| & =\left|\left(\Omega_{t} \cap\left(\Omega \backslash B_{R}(y)\right) \cup\left(\Omega_{t} \cap B_{R}(y)\right)\right)\right| \\
& =\left|\Omega_{t} \cap\left(\Omega \backslash B_{R}(y)\right)\right|+\left|\left(\Omega_{t} \cap B_{R}(y)\right)\right| .
\end{aligned}
$$

Let us consider the first part on the right-hand side of (4.12). Using (4.8) in Lemma 2 we deduce

$$
\begin{aligned}
t^{p}\left|\Omega_{t} \cap\left(\Omega \backslash B_{R}(y)\right)\right| & =\int_{\Omega_{t} \cap\left(\Omega \backslash B_{R}(y)\right)} t^{p} d t<\int_{\Omega_{t} \cap\left(\Omega \backslash B_{R}(y)\right)}\left|X G_{y}^{r}\right|^{p} d x \\
& \leq \int_{\Omega \backslash B_{R}(y)}\left|X G_{y}^{r}\right|^{p} d x \leq C R^{\frac{p-Q}{p-1}}=C t^{\frac{Q-p}{Q-1}}
\end{aligned}
$$

which implies

$$
\left|\Omega_{t} \cap\left(\Omega \backslash B_{R}(y)\right)\right| \leq C t^{\frac{Q-p}{Q-1}-p}<C t^{-\frac{(p-1) Q}{Q-1}} .
$$


Given that the assumption (H2) implies $\left|B_{R}(y)\right| \leq C R^{Q}$ for all $R>0$, we have

$$
\left|\Omega_{t} \cap B_{R}(y)\right| \leq\left|B_{R}(y)\right| \leq C R^{Q} \leq C t^{-\frac{(p-1) Q}{Q-1}} .
$$

Combining (4.13) with (4.14), we get $\left|\Omega_{t}\right| \leq C t^{-\frac{(p-1) Q}{Q-1}}$, by which we further obtain

$$
\begin{aligned}
\left\|X G^{r}\right\|_{L_{w} \frac{(p-1) Q}{Q-1}}(\Omega) & =\inf _{t>0}\left|\Omega_{t}\right| t^{\frac{(p-1) Q}{Q-1}} \\
& \leq \inf _{t>0} C\left(t^{-\frac{(p-1) Q}{Q-1}}\right) t^{\frac{(p-1) Q}{Q-1}} \\
& \leq C .
\end{aligned}
$$

(2) Let $y \in \Omega$ and $r>0$. By Property (4.2) in Theorem 7, there exists a constant $C_{1}>0$ independent of $r$ such that $\left\|G_{y}^{r}\right\|_{L_{w}^{\kappa}(\Omega)} \leq C_{1}$. Then using inequality (4.1) we have

$$
\left\|G_{y}^{r}\right\|_{L^{\nu}(\Omega)} \leq\left(\frac{\kappa}{\kappa-\nu}\right)^{\frac{1}{\nu}}|\Omega|^{\frac{1}{\nu}-\frac{1}{\kappa}}\left\|G_{y}^{r}\right\|_{L_{w}^{\kappa}(\Omega)} \leq\left(\frac{\kappa}{\kappa-\nu}\right)^{\frac{1}{\nu}}|\Omega|^{\frac{1}{\nu}-\frac{1}{\kappa}} C_{1}=C,
$$

where $\nu \in[1, \kappa)$. Analogously, by property (4.11) in part (1), provided that $r$ is sufficiently small, there exists another constant $C_{2}>0$ independent of $r$ such that $\left\|X G_{y}^{r}\right\|_{L_{w} \frac{(p-1) Q}{Q-1}(\Omega)} \leq C_{2}$. Using (4.1) and $q=\frac{(p-1) Q}{Q-1}$, we obtain

$$
\left\|X G_{y}^{r}\right\|_{L^{\nu}(\Omega)} \leq\left(\frac{q}{q-\nu}\right)^{\frac{1}{\nu}}|\Omega|^{\frac{1}{\nu}-\frac{1}{q}}\left\|X G_{y}^{r}\right\|_{L_{w}^{q}(\Omega)} \leq\left(\frac{q}{q-\nu}\right)^{\frac{1}{\nu}}|\Omega|^{\frac{1}{\nu}-\frac{1}{q}} C_{2}=C,
$$

where $\nu \in[1, q)=\left[1, \frac{(p-1) Q}{Q-1}\right)$. Therefore, we can conclude that when $r$ is sufficiently small, we have $G_{y}^{r} \in W^{1, \nu}(\Omega, X)$ with $\nu \in\left[1, \frac{(p-1) Q}{Q-1}\right)$. Because $\Omega$ is bounded and $G_{y}^{r} \in W_{0}^{1, p}(\Omega, X)$, by using Hölder's inequality and the fact that $C_{0}^{1}(\Omega)$ is dense in $W_{0}^{1, p}(\Omega, X)$, we prove that $G_{y}^{r} \in W_{0}^{1, \nu}(\Omega, X)$ with $\nu \in$ $\left[1, \frac{(p-1) Q}{Q-1}\right)$. Consequently, the proof of Theorem 9 is completed.

\section{Comparison of the Green functions}

Now we are ready to prove our comparison result, Theorem 2.

Proof of Theorem 2. Apparently, part (1) is a direct consequence of inequality (4.8) in Lemma 2.

(2) From Theorem 9 we have $G_{y}^{r} \in W_{0}^{1, \nu}(\Omega, X)$ for any $\nu \in\left[1, \frac{(p-1) Q}{Q-1}\right)$. Poincaré's inequality implies that the embedding $W_{0}^{1, \nu}(\Omega, X) \hookrightarrow L^{\nu}(\Omega)$ is compact, so it is possible to extract a subsequence $\left(G_{y}^{r_{\mu}}\right)$ such that

$$
G_{y}^{r_{\mu}} \rightarrow G_{y}(x) \text {, almost everywhere in } \Omega(\text { as } \mu \rightarrow \infty) .
$$

On the other hand, by using inequality (4.5), we get

$$
G_{y}^{r_{\mu}} \leq C d(x, y)^{\frac{p}{p-1}}\left|B_{d(x, y)}(x)\right|^{-\frac{1}{p-1}},
$$

provided that $r_{\mu}$ is sufficiently small such that $2 r_{\mu} \leq d(x, y)$, where $C=C(\lambda, Q, p$, $\Omega, X)$. By considering the limit as $\mu \rightarrow \infty$, we obtain (1.10).

(3) Let $x, y \in \Omega(x \neq y)$ satisfy $d(x, y) \leq \frac{2}{3} \operatorname{dist}(y, \partial \Omega)$. Let $\rho=d(x, y)$ and $0<R<\rho / 4$. Choose a cutoff function $\eta \in C_{0}^{\infty}\left(\Omega \backslash B_{R}\right)$ such that $0 \leq \eta \leq 1$, with 
$\eta=1$ on $B_{\rho}(y) \backslash B_{\frac{\rho}{2}}(y), \eta=0$ outside of $B_{\frac{3 \rho}{2}}(y) \backslash B_{\frac{\rho}{4}}(y)$ and $|X \eta| \leq \frac{C}{\rho}$. Taking $\phi(x)=\eta^{p} G_{y}$ as a test function in equation (1.7), we have $\mathcal{A}\left[G_{y}, \eta^{p} G_{y}\right]=0$ with $\eta=0$ on $\Omega \backslash B_{R}$. So we get

$$
\begin{aligned}
& \int_{\Omega \backslash B_{R}}\left\langle A(x) \nabla G_{y}, \nabla G_{y}\right\rangle^{\frac{p-2}{2}}\left\langle A(x) \nabla G_{y}, \nabla G_{y}\right\rangle \eta^{p} d x \\
& \quad=-p \int_{\Omega \backslash B_{R}}\left\langle A(x) \nabla G_{y}, \nabla G_{y}\right\rangle^{\frac{p-2}{2}}\left\langle A(x) \nabla G_{y}, \nabla \eta\right\rangle G_{y} \eta^{p-1} d x .
\end{aligned}
$$

According to the lower estimate on the left-hand side of (1.3) and the upper estimate on the right-hand of (1.3) and (1.4) we get

$$
\begin{aligned}
& \frac{1}{\lambda^{\frac{p}{2}}} \int_{\Omega \backslash B_{R}}\left|X G_{y}\right|^{p} \eta^{p} d x \\
& \quad \leq p \lambda^{\frac{p}{2}} \int_{\Omega \backslash B_{R}}\left|X G_{y}\right|^{p-1}|X \eta|\left|G_{y}\right| \eta^{p-1} d x \\
& \quad \leq \lambda^{\frac{p}{2}}\left(\frac{p-1}{p \lambda^{p}} \int_{\Omega \backslash B_{R}}\left|X G_{y}\right|^{p} \eta^{p} d x+\frac{1}{p \lambda^{p}} \int_{\Omega \backslash B_{R}}|X \eta|^{p} G_{y}^{p} d x\right),
\end{aligned}
$$

which implies that

$$
\int_{\Omega \backslash B_{R}}\left|X G_{y}\right|^{p} \eta^{p} d x \leq\left. C(\lambda, p) \int_{\Omega \backslash B_{R}}|X \eta|^{p}|| G_{y}\right|^{p} d x .
$$

From the assumption on $\eta$ it easily follows that

$$
\int_{\Omega \backslash B_{R}}\left|X G_{y}\right|^{p} \eta^{p} d x \geq \int_{B_{\rho}(y) \backslash B_{\frac{\rho}{2}}(y)}\left|X G_{y}\right|^{p} \eta^{p} d x=\int_{B_{\rho}(y) \backslash B_{\frac{\rho}{2}}(y)}\left|X G_{y}\right|^{p} d x .
$$

By considering that $\eta=0$ outside of $B_{\frac{3 \rho}{2}}(y) \backslash B_{\frac{\rho}{4}}(y)$ and $|X \eta| \leq \frac{C}{\rho}$, we have

$$
\begin{aligned}
\int_{\Omega \backslash B_{R}}|X \eta|^{p} G_{y}^{p} d x & =\int_{B_{\frac{3 \rho}{2}}(y) \backslash B_{\frac{\rho}{4}}(y)}|X \eta|^{p} G_{y}^{p} d x \leq C \rho^{-p} \int_{B_{\frac{3 \rho}{2}(y)} \backslash B_{\frac{\rho}{4}}(y)} G_{y}^{p} d x \\
& \leq C \rho^{-p}\left(\sup _{B_{\frac{3 \rho}{2}}(y) \backslash B_{\frac{\rho}{4}}(y)} G_{y}\right)^{p}\left|B_{\frac{3 \rho}{2}}(y)\right| .
\end{aligned}
$$

From (5.1)-(5.3), we have

$$
\int_{B_{\rho}(y) \backslash B_{\frac{\rho}{2}}(y)}\left|X G_{y}\right|^{p} d x \leq C \rho^{-p}\left|B_{\frac{3 \rho}{2}}(y)\right|\left(\sup _{B_{\frac{3 \rho}{2}}(y) \backslash B_{\frac{\rho}{4}}(y)} G_{y}\right)^{p} .
$$

We choose $\zeta$ as a cutoff function satisfying $\zeta=1$ in $B_{\frac{\rho}{2}}(y)$ and $\zeta=0$ outside of $B_{\rho}(y)$ and $|X \zeta| \leq \frac{C}{\rho}$. Let $\zeta$ be a test function. Using (1.4) and Hölder's inequality, we get

$$
\begin{aligned}
1=\mathcal{A}\left(G_{y}, \zeta\right) & \leq \lambda^{\frac{p}{2}} \int_{\Omega}\left|X G_{y}\right|^{p-1}|X \zeta| \leq \lambda^{\frac{p}{2}} C \rho^{-1} \int_{B_{\rho}(y) \backslash B_{\frac{\rho}{2}}(y)}\left|X G_{y}\right|^{p-1} d x \\
& \leq C \rho^{-1}\left|B_{\rho}(y) \backslash B_{\frac{\rho}{2}}\right|^{\frac{1}{p}}\left(\int_{B_{\rho}(y) \backslash B_{\frac{\rho}{2}}(y)}\left|X G_{y}\right|^{p} d x\right)^{\frac{p-1}{p}} \\
& \leq C \rho^{-p}\left|B_{\rho}(y) \backslash B_{\frac{\rho}{2}}\right|^{\frac{1}{p}}\left|B_{\frac{3 \rho}{2}}\right|^{\frac{p-1}{p}}\left(\sup _{B_{\frac{3 \rho}{2}}(y) \backslash B_{\frac{\rho}{4}}(y)} G_{y}\right)^{p-1} .
\end{aligned}
$$


Note that the doubling condition implies that $\left|B_{\frac{3 \rho}{2}}\right| \leq A\left|B_{\frac{3 \rho}{4}}\right| \leq A\left|B_{\rho}\right|$. By means of Harnack's inequality, the right-hand side of (5.4) becomes

$$
\begin{gathered}
C \rho^{-p}\left|B_{\rho}(y) \backslash B_{\frac{\rho}{2}}\right|^{\frac{1}{p}}\left|B_{\frac{3 \rho}{2}}\right|^{\frac{p-1}{p}}\left(\sup _{B_{\frac{3 \rho}{2}}(y) \backslash B_{\frac{\rho}{4}}(y)} G_{y}\right)^{p-1} \leq C \rho^{-p}\left|B_{\rho}\right|\left(\sup _{B_{\frac{3 \rho}{2}}(y) \backslash B_{\frac{\rho}{4}}(y)} G_{y}\right)^{p-1} \\
(5.5) \quad \leq C \rho^{-p}\left|B_{\rho}\right|\left(\inf _{B_{\frac{3 \rho}{2}}(y) \backslash B_{\frac{\rho}{4}}(y)} G_{y}\right)^{p-1} \leq C \rho^{-p}\left|B_{\rho}\right| G_{y}(z)^{p-1},
\end{gathered}
$$

where $z \in B_{\frac{3 \rho}{2}}(y) \backslash B_{\frac{\rho}{4}}(y)$. In particular, since $d(x, y)=\rho$, from (5.4) and (5.5), we deduce that $x \in B_{\frac{3 \rho}{2}}(y) \backslash B_{\frac{\rho}{4}}(y)$ and

$$
1 \leq d(x, y)^{-\frac{p}{p-1}}\left|B_{d(x, y)}(y)\right|^{\frac{1}{p-1}} G(x, y) .
$$

Since $B_{\rho}(y) \subset B_{2 \rho(y)}$, we have $\left|B_{\rho}(y)\right| \leq\left|B_{2 \rho(x)}\right| \leq\left|B_{\rho}(x)\right|$. Therefore, we can conclude that

$$
G(x, y) \geq d(x, y)^{\frac{p}{p-1}}\left|B_{d(x, y)}(x)\right|^{-\frac{1}{p-1}} .
$$

The proof of Theorem 10 is completed.

\section{Applications to nONLINEAR SUbELLIPTIC PROBlems}

In this section, we will present an important technique that the power of the Green function can be used as a kernel function so as to establish the regularity in Morrey's spaces for nonlinear subelliptic problems. We assume $x_{0} \in \Omega$ and consider the Green function $G=G\left(x, x_{0}\right)$ related to $x_{0}$, which is the solution of

$$
\int_{\Omega}\langle A(x) X u, X u\rangle^{\frac{p-2}{2}}\langle A(x) X u, X \phi\rangle d x=\phi\left(x_{0}\right), \quad \forall \phi(x) \in W_{0}^{1, p}(\Omega, X) .
$$

Suppose that $u \in W^{1, p}(\Omega, X)$ is a weak solution of the following variational problem:

$$
\int_{\Omega}\langle A(x) X u, X u\rangle^{\frac{p-2}{2}}\langle A(x) X u, X \phi(x)\rangle d x=\int_{\Omega} f(x) \phi(x) d x+\int_{\Omega}\langle g(x), X \phi(x)\rangle d x,
$$

for any $\phi(x) \in W_{0}^{1, p}(\Omega, X)$, where $f(x) \in L^{q}(\Omega)$ with $q>\frac{Q}{p}$, and the vector function $g(x) \in\left[L^{s}(\Omega)\right]^{N}$ with $s>\frac{Q}{p-1}$.

We are left with the proof of one of our main results, Theorem 3. We now do it by making use of the estimates of the Green function defined as in (6.1), the hole-filling technique, as well as the following technical lemma.

Lemma 3 ([29, Lemma 8.23]). Let $\omega$ be a nondecreasing function on the interval $\left(0, R_{0}\right]$, and for all $0<R \leq R_{0}$, which satisfies the inequality

$$
\omega(\tau R) \leq \theta \omega(R)+K R^{\alpha},
$$

where $0<\theta$ and $\tau<1$. Then, for $0<\delta<\alpha$, we have

$$
\omega(R) \leq C\left(\frac{R}{R_{0}}\right)^{\delta}\left[\omega\left(R_{0}\right)+K R_{0}^{\alpha}\right]
$$

where $C=C(\tau, \theta)$ and $\delta=\delta(\tau, \theta, \alpha)$ are positive constants.

Proof of Theorem 3. Given a point $x_{0} \in \Omega$ and a positive constant $0<R_{0}<$ $\frac{1}{4}$ dist $\left(x_{0}, \partial \Omega\right)$, we let $\eta(x)$ be a smooth cutoff function satisfying $\eta=1$ when $x \in B_{R / 2}\left(x_{0}\right), \eta=0$ when $x \in \mathbb{R}^{N} \backslash B_{2 R}\left(x_{0}\right)$, as well as $0 \leq \eta \leq 1$ with $|X \eta| \leq \frac{K}{R}$ 
for some positive constant $K$. We denote the following constant associated with $u$ and $B_{R}\left(x_{0}\right)$ by

$$
u_{R}=\frac{1}{\left|B_{2 R}-B_{R / 2}\right|} \int_{B_{2 R}-B_{R / 2}} u(x) d x, \quad \text { for } B_{2 R} \subset \Omega
$$

Letting $\psi(x)=G\left(x, x_{0}\right)^{p-1} \eta(x)^{p}$ with the Green function $G\left(x, x_{0}\right)$ defined as in (6.1), we test equation (6.1) with the test function $\phi(x)=\left(u-u_{R}\right) \psi(x)$. Since $X \phi=\psi X u+\left(u-u_{R}\right) X \psi$, we have

$$
\begin{aligned}
\int_{\Omega}\langle A(x) X u, X u\rangle^{\frac{p-2}{2}}\left\langle A(x) X u, \psi X u+\left(u-u_{R}\right) X \psi\right\rangle d x \\
\quad=\int_{\Omega} f(x)\left(u-u_{R}\right) \psi d x+\int_{\Omega}\left\langle g(x), \psi X u+\left(u-u_{R}\right) X \psi\right\rangle d x
\end{aligned}
$$

By virtue of inequalities (1.3) and (1.4) we deduce

$$
\begin{aligned}
\frac{1}{\lambda^{\frac{p}{2}}} \int_{\Omega} \psi|X u|^{p} d x \leq & \int_{\Omega} \psi\langle A(x) X u, X u\rangle^{\frac{p-2}{2}}\langle A(x) X u, X u\rangle d x \\
\leq & \int_{\Omega}\left|u-u_{R}\right|\langle A(x) X u, X u\rangle^{\frac{p-2}{2}}\langle A(x) X u, X \psi\rangle d x \\
& +\int_{\Omega}|f(x)| \cdot\left|u-u_{R}\right| \psi d x+\int_{\Omega}|g(x)| \cdot\left|\psi X u+\left(u-u_{R}\right) X \psi\right| d x \\
\leq & \lambda^{\frac{p}{2}} \int_{\Omega}\left|u-u_{R}\right||X u|^{p-1}|X \psi| d x+\int_{\Omega}|f(x)| \cdot\left|u-u_{R}\right| \psi d x \\
& +\int_{\Omega}|g(x) \cdot||X u| \psi d x+\int_{\Omega}|g(x)| \cdot\left|u-u_{R}\right||X \psi| d x \\
\equiv & I+I I,
\end{aligned}
$$

where $I=\lambda^{\frac{p}{2}} \int_{\Omega}\left|u-u_{R}\right||X u|^{p-1}|X \psi| d x$ and $I I$ represents the sum of the remaining three terms on the right-hand side of (6.2). Note that here $X \psi=p \eta^{p-1} G^{p-1} X \eta+$ $(p-1) G^{p-2} \eta^{p} X G$. After substitution of $X \psi$ into $I$, there are two terms in $I$. Using Young's inequality and Theorem 2, we can estimate the first term of $I$ as follows:

$$
\begin{aligned}
& p \lambda^{\frac{p}{2}} \int_{\Omega}\left|u-u_{R}\right||X u|^{p-1} \eta^{p-1} G^{p-1}|X \eta| d x \\
& \quad \leq K p \lambda^{\frac{p}{2}} \int_{B_{2 R}-B_{R / 2}}(\eta|X u|)^{p-1} G^{\frac{(p-1)^{2}}{p}} G^{\frac{p-1}{p}} \frac{\left|u-u_{R}\right|}{R} d x \\
& \quad \leq C \int_{B_{2 R}-B_{R / 2}}(\eta|X u|)^{p} G^{p-1} d x+C \int_{B_{2 R}-B_{R / 2}} \frac{\left|u-u_{R}\right|^{p}}{R^{p}} G^{p-1} d x \\
& \quad \leq C \int_{B_{2 R}-B_{R / 2}} \frac{|X u|^{p}}{d\left(x, x_{0}\right)^{Q-p}} d x+C \int_{B_{2 R}-B_{R / 2}} \frac{\left|u-u_{R}\right|^{p}}{R^{p} d\left(x, x_{0}\right)^{Q-p}} d x .
\end{aligned}
$$


In view of $\frac{R}{2}<d\left(x, x_{0}\right)<2 R$ in $B_{2 R}-B_{R / 2}$ and Poincaré's inequality (H3), the second part on the right-hand side of (6.3) can be expressed as

$$
\begin{aligned}
\int_{B_{2 R}-B_{R / 2}} \frac{\left|u-u_{R}\right|^{p}}{R^{p} d\left(x, x_{0}\right)^{Q-p}} d x & \leq \frac{C}{R^{p+Q-p}} \int_{B_{2 R}-B_{R / 2}}\left|u-u_{R}\right|^{p} d x \\
& \leq \frac{C}{R^{Q-p}} \int_{2\left(B_{2 R}-B_{R / 2}\right)}|X u|^{p} d x \\
& \leq C \int_{B_{4 R}-B_{R / 4}} \frac{|X u|^{p}}{d\left(x, x_{0}\right)^{Q-p}} d x
\end{aligned}
$$

Combining (6.3) and (6.4) yields

$$
\int_{\Omega}|X u|^{p-1}\left|u-u_{R}\right| \eta^{p-1} G^{p-1}|X \eta| d x \leq C \int_{B_{4 R}-B_{R / 4}} \frac{|X u|^{p}}{\left.d\left(x, x_{0}\right)\right|^{Q-p}} d x .
$$

Using Young's inequality with $\varepsilon_{0}=\frac{1}{4 \lambda^{\frac{p}{2}}}$, then the second term of $I$ becomes

$$
\begin{aligned}
& (p-1) \lambda^{\frac{p}{2}} \int_{\Omega}\left|u-u_{R}\right||X u|^{p-1} \eta^{p} G^{p-2}|X G| d x \\
& \quad=(p-1) \lambda^{\frac{p}{2}} \int_{\Omega}|\eta X u|^{p-1} G^{\frac{(p-1)^{2}}{p}} \eta\left|u-u_{R}\right| \frac{|X G|}{G^{\frac{1}{p}}} d x \\
& \quad \leq \varepsilon_{0} \int_{\Omega} G^{p-1}|\eta X u|^{p} d x+C\left(p, \lambda, \varepsilon_{0}\right) \int_{\Omega}\left|\eta\left(u-u_{R}\right)\right|^{p} \frac{|X G|^{p}}{G} d x .
\end{aligned}
$$

It remains to estimate the term

$$
\int_{\Omega}\left|\eta\left(u-u_{R}\right)\right|^{\mid} \frac{|X G|^{p}}{G} d x
$$

In order to end our proof, we take a new cutoff function $\xi(x) \in C_{0}^{\infty}(\Omega)$ with $|X \xi| \leq \frac{C}{R}$, which satisfies

$$
\xi(x)= \begin{cases}0, & \text { for } \quad d\left(x, x_{0}\right) \leq \frac{R}{4} \\ \eta, & \text { for } \quad d\left(x, x_{0}\right) \geq \frac{R}{2}\end{cases}
$$

Using the Green function $G$ defined by equation (1.7) we may take the test function as

$$
\phi(x)=G^{-\frac{1}{p}}\left[\xi(x)\left(u-u_{R}\right)\right]^{p} .
$$

Noting that $\phi\left(x_{0}\right)=0$ and

$$
X \phi=-\frac{1}{p} G^{-\frac{1+p}{p}}\left[\xi\left(u-u_{R}\right)\right]^{p} X G+p G^{-\frac{1}{p}}\left[\xi\left(u-u_{R}\right)\right]^{p-1} X\left[\xi\left(u-u_{R}\right)\right],
$$

we get

$$
\begin{aligned}
\frac{1}{p} \int_{\Omega} & \langle A(x) X G, X G\rangle^{\frac{p-2}{2}}\langle A(x) X G, X G\rangle G^{-\frac{1+p}{p}}\left[\xi\left(u-u_{R}\right)\right]^{p} d x \\
& =p \int_{\Omega}\langle A(x) X G, X G\rangle^{\frac{p-2}{2}}\left\langle A(x) X, X\left[\xi\left(u-u_{R}\right)\right]\right\rangle G^{-\frac{1}{p}}\left[\xi\left(u-u_{R}\right)\right]^{p-1} d x
\end{aligned}
$$


According to (1.3) and (1.4), and using Young's inequality with $\varepsilon_{1}=\frac{1}{4 p \lambda^{\frac{p}{2}}}$ and the above cutoff function, we deduce

$$
\begin{aligned}
\frac{1}{p \lambda^{\frac{p}{2}}} \int_{\Omega}|X G|^{p}\left|\xi\left(u-u_{R}\right)\right|^{p} G^{-\frac{p+1}{p}} d x \\
\leq p \lambda^{\frac{p}{2}} \int_{\Omega} G^{-\frac{1}{p}}|X G|^{p-1} \cdot\left|\xi\left(u-u_{R}\right)\right|^{p-1} \cdot\left|X\left[\xi\left(u-u_{R}\right)\right]\right| d x \\
\leq \varepsilon_{1} \int_{\Omega}|X G|^{p}\left|\xi\left(u-u_{R}\right)\right|^{p} G^{-\frac{p+1}{p}} d x \\
\quad+C \int_{\Omega} G^{p-1-\frac{1}{p}}\left|X\left[\xi\left(u-u_{R}\right)\right]\right|^{p} d x
\end{aligned}
$$

That is,

$$
\int_{\Omega}|X G|^{p}\left|\xi\left(u-u_{R}\right)\right|^{p} G^{-\frac{p+1}{p}} d x \leq C \int_{\Omega} G^{p-1-\frac{1}{p}}\left|\left(u-u_{R}\right) X \xi+\xi X u\right|^{p} d x .
$$

On the other hand, according to Theorem 2 and Poincaré's inequality, we have

$$
\begin{aligned}
\int_{\Omega}|X G|^{p}\left|\xi\left(u-u_{R}\right)\right|^{p} G^{-\frac{p+1}{p}} & d x \\
& \leq C \int_{B_{2 R}-B_{R / 4}} G^{p-1-\frac{1}{p}}\left(\frac{\left|u-u_{R}\right|^{p}}{R^{p}}+|X u|^{p}\right) d x \\
& =C \int_{B_{2 R}-B_{R / 4}} G^{p-1-\frac{1}{p}} \frac{\left|u-u_{R}\right|^{p}}{R^{p}} d x+C \int_{B_{2 R}-B_{R / 4}} G^{p-1-\frac{1}{p}}|X u|^{p} d x \\
& \leq C R^{p-Q-\frac{p-Q}{p(p-1)}} \int_{B_{4 R}-B_{R / 4}}|X u|^{p} d x .
\end{aligned}
$$

Since $\frac{R}{4}<d\left(x, x_{0}\right)<4 R$ for any $x \in B_{4 R}-B_{R / 4}$, we get

$$
\begin{aligned}
\int_{\Omega} \mid \xi & \left.\left(u-u_{R}\right)\right|^{p} \frac{|X G|^{p}}{G} d x \\
& \leq C R^{\frac{p-Q}{p(p-1)}} \int_{B_{4 R}-B_{R / 4}}|X G|^{p}\left|\xi\left(u-u_{R}\right)\right|^{p} G^{-\frac{p+1}{p}} d x \\
& \leq C R^{p-Q} \int_{B_{4 R}-B_{R / 4}}|X u|^{p} d x \\
& \leq C \int_{B_{4 R}-B_{R / 4}} \frac{|X u|^{p}}{d\left(x, x_{0}\right)^{Q-p}} d x .
\end{aligned}
$$

Consequently, from (6.5)-(6.8), we deduce

$$
I \leq \varepsilon_{0} \int_{\Omega} G^{p-1}|\eta X u|^{p} d x+C \int_{B_{4 R}-B_{R / 4}} \frac{|X u|^{p}}{d\left(x, x_{0}\right)^{Q-p}} .
$$

Next, we show that the second item $I I$ in (6.2) satisfies

$$
\begin{aligned}
& I I \equiv \int_{\Omega}|f(x)| \cdot\left|u-u_{R}\right| \psi d x+\int_{\Omega}|g(x)| \cdot|X u| \psi d x+\int_{\Omega}|g(x)| \cdot\left|u-u_{R}\right||X \psi| d x \\
& \quad \leq\left(\varepsilon_{2}+\varepsilon_{3}+\varepsilon_{4}\right) C \int_{B_{R / 4}} \frac{|X u|^{p}}{d\left(x, x_{0}\right)^{Q-p}} d x+C \int_{B_{4 R}-B_{R / 4}} \frac{|X u|^{p}}{d\left(x, x_{0}\right)^{Q-p}} d x+C R^{\vartheta_{0}},
\end{aligned}
$$


where $\vartheta_{0}$ is a positive constant and $\varepsilon_{k}(k=2,3,4)$ are small positive constants. On the basis of Young's inequality with $\varepsilon_{2}>0$ and Poincaré's inequality, the first term of the right-hand side in (6.10) satisfies

$$
\begin{aligned}
\int_{\Omega}|f(x)| \cdot\left|u-u_{R}\right| G^{p-1} \eta^{p} d x \\
\leq C\left(\varepsilon_{2}\right) \int_{B_{2 R}}|f(x) R|^{\frac{p}{p-1}} G^{p-1} d x+\varepsilon_{2} \int_{B_{2 R}} \frac{\left|u-u_{R}\right|^{p}}{R^{p}} G^{p-1} d x \\
\leq C \int_{B_{2 R}}|R f(x)|^{\frac{p}{p-1}} G^{p-1} d x+\varepsilon_{2} C \int_{B_{4 R}} \frac{|X u|^{p}}{d\left(x, x_{0}\right)^{Q-p}} d x \\
\leq \varepsilon_{2} C \int_{B_{R / 4}} \frac{|X u|^{p}}{d\left(x, x_{0}\right)^{Q-p}} d x+C \int_{B_{4 R}-B_{R / 4}} \frac{|X u|^{p}}{d\left(x, x_{0}\right)^{Q-p}} d x \\
\quad+C \int_{B_{2 R}}|R f(x)|^{\frac{p}{p-1}} G^{p-1} d x .
\end{aligned}
$$

In view of estimates of the Green function in Theorem 2, by Hölder's inequality, the third term on the right-hand side of (6.11) becomes

$$
\begin{aligned}
\int_{B_{2 R}} & |R f(x)|^{\frac{p}{p-1}} G^{p-1} d x \\
\leq C R^{\frac{p}{p-1}} & \int_{B_{2 R}} d\left(x, x_{0}\right)^{p-Q}|f(x)|^{\frac{p}{p-1}} d x \\
& \leq C R^{\frac{p}{p-1}+p-Q} \int_{B_{2 R}}|f(x)|^{\frac{p}{p-1}} d x \\
& \leq C R^{\frac{p}{p-1}+p-Q}\left|B_{2 R}\right|^{1-\frac{p}{(p-1) q}}\|f(x)\|_{L^{q}}^{\frac{p}{p-1}} \\
& \leq C R^{p+\frac{p(q-Q)}{(p-1) q}}\|f(x)\|_{L^{\frac{p}{p-1}}} .
\end{aligned}
$$

It is easy to see that $p+\frac{p(q-Q)}{(p-1) q}>0$ due to the hypothesis of $q>\frac{Q}{p}$.

Similarly, by Young's inequality again, the second part and the third part of $I I$, respectively, satisfy

$$
\begin{aligned}
& \int_{\Omega}|g(x)| \cdot|X u| G^{p-1} \eta^{p} d x \\
& \leq C \int_{B_{2 R}}|g(x)|^{\frac{p}{p-1}} G^{p-1} d x+\varepsilon_{3} \int_{B_{2 R}}|X u|^{p} G^{p-1} d x \\
& \leq \varepsilon_{3} C \int_{B_{R / 4}} \frac{|X u|^{p}}{d\left(x, x_{0}\right)^{Q-p}} d x \\
&+C \int_{B_{4 R}-B_{R / 4}} \frac{|X u|^{p}}{d\left(x, x_{0}\right)^{Q-p}} d x+C \int_{B_{2 R}}|g(x)|^{\frac{p}{p-1}} G^{p-1} d x \\
& \leq \varepsilon_{3} C \int_{B_{R / 4}} \frac{|X u|^{p}}{d\left(x, x_{0}\right)^{Q-p}} d x \\
&+C \int_{B_{4 R}-B_{R / 4}} \frac{|X u|^{p}}{d\left(x, x_{0}\right)^{Q-p}} d x+C R^{p-\frac{p Q}{(p-1) s}}\|g(x)\|_{L^{s}}^{\frac{p}{p-1}}
\end{aligned}
$$


and

$$
\begin{aligned}
& \int_{\Omega}|g(x)| \cdot\left|u-u_{R}\right|\left|p \eta^{p-1} G^{p-1} X \eta\right| d x \\
& \quad \leq C \int_{B_{2 R}-B_{R / 2}}|g(x)|^{\frac{p}{p-1}} G^{p-1} d x+C \int_{B_{2 R}-B_{R / 2}} \frac{\left|u-u_{R}\right|^{p}}{R^{p}} G^{p-1} d x \\
& \quad \leq C \int_{B_{2 R}-B_{R / 2}}|g(x)|^{\frac{p}{p-1}} G^{p-1} d x+\int_{B_{4 R}-B_{R / 4}} \frac{|X u|^{p}}{d\left(x, x_{0}\right)^{Q-p}} d x \\
& \quad \leq \int_{B_{4 R}-B_{R / 4}} \frac{|X u|^{p}}{d\left(x, x_{0}\right)^{Q-p}} d x++C R^{p-\frac{p Q}{(p-1) s}}\|g(x)\|_{L^{s}}^{\frac{p}{p-1}} .
\end{aligned}
$$

Furthermore, by using Young's inequality and (6.8), we deduce

$$
\begin{aligned}
& \int_{\Omega}|g(x)| \cdot\left|u-u_{R}\right| \cdot\left|(p-1) G^{p-2} X G\right| \eta^{p} d x \\
&=(p-1) \int_{\Omega}|g(x)| G^{\frac{(p-1)^{2}}{p}} \eta^{p} \frac{\left|u-u_{R}\right||X G|}{G^{\frac{1}{p}}} d x \\
& \leq C \int_{B_{2 R}}|g(x)|^{\frac{p}{p-1}} G^{p-1} \eta^{p} d x+\varepsilon_{4} \int_{B_{2 R}}\left|\eta\left(u-u_{R}\right)\right|^{p} \frac{|X G|^{p}}{G} d x \\
& \leq C \int_{B_{2 R}}|g(x)|^{\frac{p}{p-1}} G^{p-1} d x+\varepsilon_{4} C \int_{B_{4 R}}|X u|^{p} d\left(x, x_{0}\right)^{p-Q} d x \\
& \leq \varepsilon_{4} C \int_{B_{R / 4}}|X u|^{p} d\left(x, x_{0}\right)^{p-Q} d x+C \int_{B_{4 R}-B_{R / 4}}|X u|^{p} d\left(x, x_{0}\right)^{p-Q} d x \\
&+C R^{p-\frac{p Q}{(p-1) s}}\|g(x)\|_{L^{s}}^{\frac{p}{p-1}}
\end{aligned}
$$

where it is easy to see that $p-\frac{Q p}{(p-1) s}>0$ due to the hypothesis of $s>\frac{Q}{p-1}$.

We then choose

$$
\vartheta_{0}=\min \left\{1+\frac{(q-Q)}{(p-1) q}, 1-\frac{Q}{(p-1) s}\right\},
$$

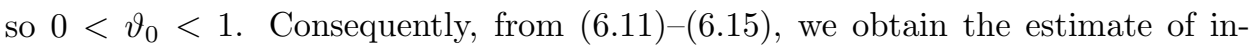
equality (6.10).

Now, let us substitute (6.9) and (6.10) into (6.2) and take small positive constants for $\varepsilon_{k}(k=2,3,4)$ such that $\left(\varepsilon_{2}+\varepsilon_{3}+\varepsilon_{4}\right) C<\frac{1}{4 \lambda^{\frac{p}{2}}}$. Then we can obtain the estimate with some positive constants $K_{0}$ and $C$ which only depend on $\lambda, Q, p$ and $X$ as

$$
\int_{B_{R / 4}} \frac{|X u|^{p}}{d\left(x, x_{0}\right)^{Q-p}} d x \leq K_{0} \int_{B_{4 R}-B_{R / 4}} \frac{|X u|^{p}}{\left|d\left(x, x_{0}\right)\right|^{Q-p}} d x+C R^{p \vartheta_{0}} .
$$

Fixing the value of $R_{0}$ with $0<R_{0}<\frac{1}{4}$ dist $\left(x_{0}, \partial \Omega\right)$, due to $X u \in L^{p}(\Omega)$ and $\frac{R_{0}}{4}<d\left(x, x_{0}\right)<4 R_{0}$ in $B_{4 R_{0}}-B_{R_{0} / 4}$, we have

$$
\int_{B_{R_{0} / 4}} \frac{|X u|^{p}}{d\left(x, x_{0}\right)^{Q-p}} d x \leq \frac{C}{R_{0}^{Q-p}}\|X u\|_{L^{p}(\Omega)}^{p}+C R_{0}^{p \vartheta_{0}}<+\infty
$$

or

$$
\int_{B_{4 R_{0}}} \frac{|X u|^{p}}{d\left(x, x_{0}\right)^{Q-p}} d x<+\infty
$$


On the other hand, from (6.16) we find

$$
\int_{B_{R / 4}} \frac{|X u|^{p}}{d\left(x, x_{0}\right)^{Q-p}} d x \leq \frac{K_{0}}{K_{0}+1} \int_{B_{4 R}} \frac{|X u|^{p}}{d\left(x, x_{0}\right)^{Q-p}} d x+C R^{p \vartheta_{0}} .
$$

Since $0<\frac{K_{0}}{K_{0}+1}<1$, by virtue of Lemma 3 we obtain

$$
\int_{B_{R}} \frac{|X u|^{p}}{d\left(x, x_{0}\right)^{Q-p}} d x \leq C R^{p \vartheta}
$$

i.e.,

$$
\int_{B_{R}}|X u|^{p} d x \leq C R^{Q-p+p \vartheta} .
$$

Therefore, we obtain that $X u \in L_{l o c}^{p, \lambda}(\Omega)$ for every $0<\lambda \leq Q-p+p \vartheta$ with

$$
\vartheta=\min \left\{\vartheta_{0}, \log _{2} \frac{1+K_{0}}{K_{0}}\right\}>0
$$

This implies that the proof of Theorem 3 has been completed.

We now define

$$
S^{0, \alpha}(\Omega)=\left\{f \in L^{\infty}(\Omega):[f]_{\alpha, \Omega}^{X}=\sup _{x, y \in \Omega} \frac{|f(x)-f(y)|}{d(x, y)^{\alpha}}<+\infty\right\} .
$$

Certainly, we can locally define the spaces $S_{l o c}^{0, \alpha}(\Omega)$ as usual.

Lemma 4 (Morrey's type lemma). Let $u \in W^{1, p}(\Omega, X)$. If there exists a constant $M>0$ such that

$$
\int_{B_{R}\left(x_{0}\right)}|X u|^{p} d x \leq M R^{Q-p+p \gamma}, \quad \forall B_{2 R}\left(x_{0}\right) \in \Omega,
$$

for some $\gamma \in(0,1)$, then $u \in S_{\text {loc }}^{0, \gamma}(\Omega)$.

Proof of Corollary 1. Now we can use Lemma 4 to prove Corollary 1 directly. On the basis of the hypothesis (H3) of Poincaré's inequality, it follows that

$$
\int_{B_{R}(x)}\left|u-u_{x, R}\right|^{2} d y \leq C R^{2} \int_{B_{R}(x)}|X u|^{2} d y \leq M R^{Q+2 \vartheta} .
$$

So we have that $u \in \mathcal{L}_{\text {loc }}^{2, Q+2 \vartheta}(\Omega)$, where $\mathcal{L}^{2, \lambda}$ is Campanato's space. Because of the equivalence of Campanato's space $\mathcal{L}^{2, \lambda}(Q<\lambda<Q+2)$ and Hölder's space $S^{0, \frac{\lambda-Q}{2}}$ [29, 25], we obtain that $u \in S^{0, \vartheta}\left(\Omega^{\prime}, X\right)$ for any $\Omega^{\prime} \Subset \Omega$. The proof of Corollary 1 is completed.

Therefore, Corollary 1 is a direct conclusion of Theorem 3.

\section{Conclusion}

Studying the Green functions for various differential systems has been an important subject in the community of differential equations and dynamical systems in the last century. We know that for a linear differential equation, the Green function is the kernel of the integral operator inverse to the differential operator generated by the given differential equation and the homogeneous boundary conditions. But for nonlinear differential equations or operators under various conditions, the analysis will become more complicated and challenging. In this work, we were concerned with the generalized Green function of regular Laplacian operators for a class of 
degenerate operators with X-ellipticity. The proofs of our main results do not request the Hörmander condition. Under certain X-elliptic conditions in the sense of distributions, we presented a maximum principle and some a priori estimates of degenerate equations. A local comparison of the generalized Green function with its fundamental solutions was established. Finally, as an application, by means of the power of Green functions as kernel functions for local integrals we obtained local Hölder continuity for nonlinear degenerate subelliptic equations.

\section{ACKNOWLEDGEMENTS}

The authors sincerely thank one anonymous referee for his valuable and constructive comments. The first author is also grateful to the Chern Institute of Mathematics, Nankai University, Tianjin, China, for generous support and hospitality when he was a visiting professor in September of 2007.

\section{REFERENCES}

[1] Bensoussan A. and Frehse J., Regularity results for nonlinear elliptic systems and applications, Applied Math. Sci., Vol. 151, Springer-Verlag, 2002. MR.1917320 (2004a:35001)

[2] Bonfigliol A., Lanconelli E. and Uguzzoni F., Stratified Lie groups and potential theory for their sub-Laplacians, Springer Monographs in Mathematics, Springer, Berlin, 2007. MR2363343 (2009m:22012)

[3] Bellaiche A. and Risler J. J., Sub-Riemannian geometry, Birkhäuser Verlag, Boston, 1996. MR.1421821 (97f:53002)

[4] Capogna L., Subelliptic mollifiers and a basic pointwise estimate of Poincaré type, Math. Z. 226 (1) (1997) 147-154. MR1472145 (98i:35025)

[5] Capogna L., Pointwise Schauder estimates for second order linear equations in Carnot groups, Harmonic Analysis at Mount Holyoke, 320 (2003) 45-69. MR.1979931 (2004m:35029)

[6] Capogna L., Danielli D. and Garofalo N., An embedding theorem and the Harnack inequality for nonlinear subelliptic equations, Comm. Part. Diff. Equs. 18 (1993) 1765-1794. MR:1239930 (94j:35038)

[7] Capogna L., Danielli D. and Garofalo N., Capacitary estimates and the local behavior of solutions of nonlinear subelliptic equations, Amer. J. Math. 118 (1997) 1153-1196. MR.1420920 (97k:35033)

[8] Chanillo S. and Wheeden R. L., Existence and estimates of Green's function for degenerate elliptic equations, Ann. Scuola Norm. Sup. Pisa 15 (1998) 309-340. MR1007400 (91b:35045)

[9] Chen G., Li Y., Zhu X. and Cao D.M., Advances in Nonlinear Partial Differential Equations and Related Areas (Beijing, 1997), World Sci. Publ., River Edge, NJ, 1998. MR1690817 (99m:35002)

[10] Citti G., Garofalo N. and Lanconelli E., Harnack's inequality for sum of squares of vector fields plus a potential, Amer. J. Math. 115 (1993) 699-734. MR.1221840 (94m:35069)

[11] Citti G. and Manfredini M., Implicit function theorem in Carnot-Carathéodory spaces, Comm. Contemp. Math. 8 (2006) 657-680. MR2263950 (2007k:53031)

[12] Di Fazio G., Domokos A., Fanciullo M. S. and Manfredi J. J., Subelliptic Cordes estimates in the Grusin plane, Manuscripta Math. 120 (4) (2006) 419-433. MR.2245893 (2008e:35027)

[13] Danielli D. and Garofalo N., Geometric properties of solutions to subelliptic equations in nilpotent Lie groups, Reaction Diffusion Systems, 194 (1998) 89-105. MR1472512|(99a:22015)

[14] Di Fazio G., Palagachev D. K. and Ragusa M. A., On Morrey's regularity of strong solutions to elliptic boundary value problems, C. R. Acad. Bulgare Sci. 50 (11-12) (1997) 17-20. MR 1709471

[15] Di Fazio G., Palagachev D. K. and Ragusa M. A., Global Morrey regularity of strong solutions to the Dirichlet problem for elliptic equations with discontinuous coefficients, J. Funct. Anal. 166 (2) (1999) 179-196. MR1707751 (2000d:35037)

[16] Di Fazio G. and Zamboni P., Unique continuation of non negative solutions to quasilinear subelliptic equations in Carnot Carathéodory spaces, Comm. Appl. Nonlinear Anal. 10 (2) (2003) 97-105. MR1992310 (2004g:35048) 
[17] Di Fazio G. and Zamboni P., Hölder continuity for quasilinear subelliptic equations in CarnotCarathéodory spaces, Math. Nachr. 272 (2004) 3-10. MR2079757 (2006a:35039)

[18] Ferrari F., A local doubling formula for the harmonic measure associated with subelliptic operators and applications, Comm. Part. Diff. Equs. 28 (1-2) (2003) 1-60. MR.1974448 (2004g:35050)

[19] Ferrari F., Harnack inequality for two-weight subelliptic $p$-Laplace operators, Math. Nach. 279 (8) (2006) 815-830. MR2228656 (2007b:35061)

[20] Franchi B., Gutiérrez C. E. and Wheeden R. L., Weight Sobolev-Poincaré inequalities for Grushin type operators, Comm. Part. Diff. Equs. 19 (3-4) (1994) 523-604. MR.1265808 (96h:26019)

[21] Franchi B. and Lanconelli E., Une métrique associée à une classe d'opérateurs elliptiques dégénérés (in French), Conference on linear partial and pseudo-differential operators (Torino, 1982), Rend. Sem. Mat. Univ. Politec. Torino 1983, Special Issue, (1984) 105-114. MR745979 (86d:35057)

[22] Franchi B. and Lanconelli E., An embedding theorem for Sobolev spaces related to nonsmooth vector fields and Harnack inequality, Comm. Part. Diff. Equs. 9 (13) (1984) 12371264. MR764663 (86b:46048)

[23] Folland G. B., Subelliptic estimates and function spaces on nilpotent Lie groups, Ark. Mat. 13 (2) (1975) 161-207. MR0494315 (58:13215)

[24] Fefferman C. and Phong D. H., Subelliptic eigenvalue problems, Proceedings of Conference on Harmonic Analysis, in honor of Antoni Zygmund, Vols. I-II, 590-606, Wadsworth Math. Ser., Wadsworth, Belmont, CA, 1983. MR730094 (86c:35112)

[25] Giaquinta M., Multiple Integrals in the Calculus of Variations and Nonlinear Elliptic Systems, Princeton University Press, Ann. Math. Stud. Vol. 105, 1983. MR.717034 (86b:49003)

[26] Gutiérrez C. E. and Lanconelli E. W., Maximum principle, non-homogeneous Harnack inequality, and Liouville theorems for X-elliptic operators, Comm. Part. Diff. Equs. 28 (11-12) (2003) 1833-1862. MR2015404(2004j:35030)

[27] Garofalo N. and Nhieu D. M., Isoperimetric and Sobolev inequalities for Carnot-Carathéodory spaces and the existence of minimal surfaces, Comm. Pure Appl. Math. 49 (1996) 1081-1144. MR.1404326 (97i:58032)

[28] Garofalo N. and Nhieu D. M., Lipschitz continuity, global smooth approximation and extension theorems for Sobolev functions in Carnot-Carathéodory spaces, J. Analyse Math. 74 (1998) 67-97. MR1631642 (2000i:46025)

[29] Gilbarg D. and Trudinger N. S., Elliptic partial differential equations of second order, 2nd ed. Spinger-Verlag, New York, 1983. MR:737190 (86c:35035)

[30] Grüter M. and Widman K., The Green function for uniformly elliptic equations, Manuscripta Math. 37 (1982) 303-342. MR657523 (83h:35033)

[31] Hayslaz P. and Koskela P., Sobolev met Poincaré, Mem. Amer. Math. Soc. 145 (2000), no. $688, \mathrm{x}+101$ pp. MR.1683160 (2000j:46063)

[32] Heinonen J., Kilpeläinen T. and Martio O., Nonlinear potential theory of degenerate elliptic equations, Clarendon Press, 1993. MR.1207810(94e:31003)

[33] Hörmander L., Hypoelliptic second order differential equations, Acta Math. 119 (1967) 147171. MR.0222474 (36:5526)

[34] Jerison D. and Lee J. M., Extremals for the Sobolev inequality on the Heisenberg group and the CR Yamabe problem, J. Amer. Math. Soc. 1 (1) (1988) 1-13. MR924699 (89b:53063)

[35] Jerison D. and Lee J. M.: Intrinsic CR coordinate and CR Yamabe problem, J. Diff. Geom. 29 (2) (1989) 303-343. MR.982177 (90h:58083)

[36] Kogoj A. E. and Lanconelli E., Liouville theorem for $X$-elliptic operators, Nonlinear Anal. 70 (8) (2009) 2974-2985. MR2509383 (2010e:35055)

[37] Kilpeläinen T. and Malý J., The Wiener test and potential estimates for quasilinear elliptic equations, Acta Math. 172 (1994) 137-161. MR.1264000 (95a:35050)

[38] Koshelev A. K., Regularity problem for quasilinear elliptic and parabolic systems, SpringerVerlag, Berlin, 1995. MR1442954 (98j:35004)

[39] Li Y., Interior gradient estimates for solutions of certain fully nonlinear elliptic equations, J. Diff. Equs. 90 (1991) 172-185. MR1094454 (92a:35071)

[40] Lanconelli E. and Kogoj A. E., $X$-elliptic operators and $X$-control distances, Contributions in honor of the memory of Ennio De Giorgi, Ricerche Mat. (suppl.) 49 (2000) 223-243. MR1826225 (2002c:35121) 
[41] Lindqvist P. and Martio O., Two theorems of N. Wiener for quasilinear elliptic equations, Acta Math. 155 (1985) 153-171. MR806413 (87g:35074)

[42] Littman W., Stampacchia G. and Weinberger H., Regular points for elliptic equations with discontinuous coefficients, Ann. Scuola Normale Superiore di Pisa (III), 17 (1963) 45-79. MR0161019 (28:4228)

[43] Mazzoni G., Green function for X-elliptic operators, Manuscripta Math. 115 (2004) 207-238. MR2098471 (2007b:35063)

[44] Manfredi J. J. and Mingione G., Regularity results for quasilinear elliptic equations in the Heisenberg group, Math. Ann. 339 (2007) 485-544. MR2336058 (2008k:35063)

[45] Negrini P. and Scornazzani V., Wiener criterion for a class of degenerate elliptic operators, J. Diff. Equs. 66 (2) (1987) 151-164. MR871992 (88b:35081)

[46] Rothschild L. P. and Stein E. M., Hypoelliptic differential operators and nilpotent groups, Acta Math. 138 (1976) 247-320. MR0436223 (55:9171)

[47] Trudinger N. S. and Wang X. J., On the weak continuity of elliptic operators and applications to potential theory, Amer. J. Math. 124 (2002) 369-410. MR1890997/(2003c:35025)

[48] Xu C. J. and Zuily C., Higher interior regularity for quasilinear subelliptic systems, Calc. Var. 5 (1997) 323-343. MR1450714 (98e:35039)

[49] Zheng S.Z. and Feng Z., Regularity for quasi-linear elliptic systems with discontinuous coefficients, Dyn. Partial Differ. Equs. 5 (2008) 87-99. MR2397307 (2009b:35084)

[50] Zheng S.Z., Zheng X. L. and Feng Z., Regularity for a class of degenerate elliptic equations with discontinuous coefficients under natural growth, J. Math. Anal. Appl. 346 (2008) 359373. MR2431532(2010a:35087)

Department of Mathematics, Beijing Jiaotong University, Beijing 100044, People's Republic of China

E-mail address: shzhzheng@bjtu.edu.cn

Department of Mathematics, University of Texas-Pan American, Edinburg, Texas 78539

E-mail address: zsfeng@utpa.edu 